\title{
Learning and Complementarities in Speculative Attacks*
}

\author{
Itay Goldstein, Emre Ozdenoren, and Kathy Yuan ${ }^{\dagger}$
}

September 2008

\begin{abstract}
We study a model where the aggregate trading of currency speculators reveals new information to the central bank and affects its policy decision. We show that the learning process gives rise to coordination motives among speculators leading to large currency attacks that cannot be explained by fundamentals and introducing nonfundamental volatility into exchange rates and policy decisions. We derive comparative statics regarding the nature of currency attacks and the effectiveness of the central bank's decision. We show that the central bank can improve the ex-ante effectiveness of its policy by committing to put a lower weight ex-post on the information coming from the market, and that transparency may either increase or decrease the effectiveness of learning from the market, depending on how it is implemented.
\end{abstract}

Journal of Economic Literature Classification Codes: G14, G15, F31, C7.

Keywords: Currency attacks, financial markets, global games, strategic complementarities, heterogenous information, learning, coordination, feedback effect..

${ }^{*}$ We thank Franklin Allen, George-Marios Angeletos, Rossella Argenziano, Sohnke Bartam, Tilman Börgers, Amil Dasgupta, Joao Gomes, Peter Kondor, Gregor Matvos, Stephen Morris, Alessandro Pavan, Lones Smith, Avindhar Subrahmanyam, and seminar and conference participants at the AFA 2008 Annual Meeting, Boston College, the International Finance Conference at Queen's University, Johns Hopkins University, London Business School, London School of Economics, the Stony Brook Workshop on Global Games, the University of Chicago "Beyond Liquidity" Conference, the University of Michigan, the University of Pennsylvania, and the University of Toronto for their many insightful comments.

${ }^{\dagger}$ Goldstein is from the Department of Finance, Wharton School, University of Pennsylvania. E-mail: itayg@wharton.upenn.edu. Ozdenoren is from the Department of Economics, University of Michigan. Email: emreo@umich.edu. Yuan is from the Department of Finance, Ross School, University of Michigan. E-mail: kyuan@umich.edu 


\section{Introduction}

By aggregating the heterogenous beliefs possessed by many speculators, currency markets, like other financial markets, provide useful information about economic fundamentals. It is therefore not surprising that central banks, like other decision makers, pay close attention to the market, trying to extract new information that will guide their decision process. Indeed, in a related context, Piazzesi (2005) provides evidence that monetary policy is affected by market data. ${ }^{1}$

In this paper, we study a model where the central bank learns from the speculative trading in currency markets about the viability of its currency regime, and uses the inferred information to guide its policy decisions. While learning from the market is usually perceived as a positive step helping to improve the decision process of the central bank, our theoretical analysis uncovers a surprising result. The very fact that the central bank learns from the market turns out to facilitate coordination motives among speculators leading to large currency attacks that introduce non-fundamental volatility into exchange rates and policy making. Our model thus proposes a mechanism that explains the currency attacks observed in many episodes, such as the collapse of the European Exchange Rate Mechanism (ERM) and the Asian financial crisis.

Traditional models explain currency attacks as a run on the foreign reserves of the central bank (see Salant and Henderson (1978), Krugman (1979), Flood and Garber (1984), Obstfeld (1996), and Morris and Shin (1998)). Our information-based approach offers empirical predictions that are distinct from those coming out of the traditional approach and that can be directly taken to the data. We describe those predictions later. At a more basic level, our approach offers the following two advantages relative to the traditional approach.

First, the relevance that foreign reserves have for the government's decision to abandon a fixed exchange rate regime is often challenged on both theoretical and empirical grounds. As Drazen (2000) summarizes: "Theoretically, low reserves is neither a necessary condition for a collapse, as the peg can be abandoned even though reserves are adequate, nor is it a sufficient condition, as reserves can be borrowed. Empirically, the collapse of a fixed exchange rate often occurs well before reserves, or some other trigger variable, hits a critical level." Moreover, the traditional approach is suited to explain only speculative attacks against an

\footnotetext{
${ }^{1}$ In another context, Baker, Stein, and Wurgler (2003), Luo (2005), and Chen, Goldstein, and Jiang (2007) show that financial-market prices affect firms' real investments.
} 
over-appreciated currency, since reserves do not pose a problem when the currency is overdepreciated. Our approach, on the other hand, can be used to analyze attacks against an over-depreciated currency, such as the Chinese Yuan, which has come under several rounds of attacks in recent years, some of them leading to significant revaluations.

Second, learning by the central bank from market outcomes is a natural channel to explore in the context of exchange rate policy. Empirical research by Ito, Lyons, and Melvin (1998), Lyons (2001), and Evans and Lyons (2002) demonstrates the importance of private and heterogenous information in currency trading. To the extent that the central bank is not fully informed about the state of the economy, it is expected that the central bank will try to infer some of this information from the market. ${ }^{2}$ For example, Burnside, Eichenbaum, and Rebelo (2001) argue that the implicit bailout guarantees to failing banks played an important role in the Asian crisis. Relating this to our paper, the currency market may aggregate market participants' heterogeneous beliefs about the state of the banking system, and the central bank may try to infer this information from the market. As mentioned above, in a related context, Piazzesi (2005) documents that the Federal Reserve Bank in the U.S. indeed incorporates the information in bond prices in its monetary policy. ${ }^{3}$

Turning to the details of our model, we analyze a situation where a central bank makes a decision whether to maintain a previously-announced fixed exchange rate regime. The central bank wants to maintain the regime only if the fundamentals of the economy are strong enough to support it. The central bank is only partially informed about the fundamentals of the economy. Speculators in the currency market also have pieces of information, which they use when deciding whether to speculate against the currency regime. By observing speculators' activities, the central bank gets an aggregate picture of the pieces of information held by speculators. Seeing a large attack against the currency, the central bank may come to believe

\footnotetext{
${ }^{2}$ The notion of heterogenous and private information is slowly being introduced into models of currency markets. See, for example, Morris and Shin (1998), Bacchetta and van Wincoop (2006), Angeletos and Werning (2006), Hellwig, Mukherji, and Tsyvinski (2006), Angeletos, Hellwig, and Pavan (2007), and Broner (2008). None of these papers, however, considers learning by the central bank.

${ }^{3}$ It is important to note that the notion that central banks are fully informed before a speculative attack erupts seems inconsistent with anecdotal evidence from real-world episodes. In these episodes, it is often the case that a central bank defends the currency regime for a period of time, and then abandons it. Our approach rationalizes this phenomenon by introducing learning by the central bank about the fundamentals of the regime. In parallel and independent work, Kurlat (2008) provides a model that addresses this concern by assuming that the central bank learns from the currency attack about the types of speculators in the market.
} 
that the fundamentals are bad and thus that it should abandon the regime. ${ }^{4}$

Coordination motives arise in this framework because speculators know that a large speculative attack against the regime has the potential of convincing the central bank that the fundamentals are weak and that the regime should be abandoned. Hence, the expectation of a large attack increases the incentive of an individual speculator to speculate against the regime. Importantly, strategic complementarities in our model arise endogenously as a result of the learning by the central bank and are not imposed on the payoff functions like in the existing literature (e.g., Morris and Shin (1998)). Hence, we distinguish the informational complementarities in our framework from the direct complementarities in the previous literature on currency attacks.

Because of the presence of complementarities, speculators put too much weight on their correlated sources of information and too little weight on their idiosyncratic sources of information when deciding whether to speculate against the currency. This implies that noise in the speculators' correlated signals gets to have a large impact on aggregate market outcomes and on the central bank's policy decision, which introduces non-fundamental volatility into exchange rates and regime shifts. This rationalizes the empirical results documented by Eichengreen, Rose, and Wyplosz (1995) and others, showing that there are excessive transitions between exchange rate regimes and that these transitions cannot be explained by fundamentals. The irony is that learning from the market by the central bank is selfdefeating. This is because the learning process is the source of the complementarities that end up reducing the informativeness of the market outcome.

Our analysis shows that the informational complementarities in our paper generate quite different implications from the usual direct complementarities. For example, while a decrease in the transaction cost of attacking the currency increases the probability that speculators will participate in a speculative attack, it has no effect on the probability that the central bank will abandon the regime. This is because the central bank cares only about the informational content of the attack, and thus filters out the effect that transaction costs have on its size. This implies that while some speculative attacks will be defended, others that are even weaker (but more informative) will not be defended.

Based on this logic, variables that characterize the quality of information turn out to have the most substantial effect in our model. When the idiosyncratic sources of the speculators'

\footnotetext{
${ }^{4}$ The regime change decision in the model can be interpreted more broadly to capture other central bank policy decisions such as intervention in a managed-float exchange rate environment.
} 
information become more precise, the quality of the information inferred by the central bank from the trading outcome increases and the central bank becomes more likely to make a decision that is justified by fundamentals. On the other hand, the precision of the common element in the speculators' information sets may have the opposite effect, as an increase in the precision of this source of information may increase the ability of speculators to coordinate on misleading the central bank into taking the wrong action. ${ }^{5}$ As we explain in the paper, given the importance of the informativeness of the trading process, potential tests of our model can be based on market microstructure measures as in Easley, Hvidkjaer, and O'Hara (Easley, Hvidkjaer, and O'Hara (2002)).

Finally, we study the desirability of two policy tools in our framework. First, we show that the central bank can improve the ex-ante effectiveness of learning from the market by committing to put a lower weight on the information conveyed by the speculative attack than is ex-post efficient. While this generates more errors ex-post, it changes the ex-ante incentives of speculators in a way that makes them put a lower weight on their common information. This, in turn, increases the informativeness of their aggregate action. Hence, the central bank is exposed to a time-inconsistency problem in its exchange rate policy. The ability to commit is therefore crucial to ensure better policy decisions.

Second, we analyze the effects of central bank transparency. ${ }^{6}$ We find that the impact of transparency is quite delicate. We discuss two related notions of transparency. In the first, speculators commonly observe a noisy signal of the central bank's information. We show that this reduces policy effectiveness because it provides common information to the speculators about the action that the central bank is likely to take, and this enables the speculators to coordinate on their common information more effectively. In the second notion, speculators heterogeneously interpret the central bank's communication. In this case, more transparency is always good, because giving speculators better heterogeneous information reduces their ability to coordinate and leads them to reveal more accurate information through the attack.

Relating the mechanism in our model to the broader literature, we note that informational externalities are common in models of financial markets. Usually in these models, strategic

\footnotetext{
${ }^{5}$ The decrease in efficiency following an increase in the precision of a public signal is reminiscent of the result in Morris and Shin (2002) and Angeletos and Pavan (2007), which was obtained in a framework with direct complementarities.

${ }^{6}$ There is a large literature on transparency. Some recent works include Morris and Shin (2002, 2005), Heinemann and Cornand (2004), Woodford (2005), Svensson (2005), Hellwig (2005), and Angeletos and Pavan (2007).
} 
substitutes arise among speculators, as the information that motivates a speculator to trade gets reflected in the price and discourages other speculators from trading or from acquiring information (see, e.g., Grossman and Stiglitz (1980)). Strategic complementarities are less common in such models, and may result from the assumption that agents have short horizons (e.g., Froot, Scharfstein, and Stein (1992), and Allen, Morris, and Shin (2006)) or from special assumptions on the distribution of noise or fundamentals (e.g., Barlevy and Veronesi (2000)). In our model, on the other hand, strategic complementarities among currency speculators arise solely due to the feedback effect that the information in their trades has on the policy decision of the policy maker.

Focusing on this feedback effect, our paper is related to a small, but growing, branch of models in financial economics that consider the feedback effect from trading in financial markets to corporate investments. Earlier contributions to this literature include Fishman and Hagerty (1992); Leland (1992); Khanna, Slezak, and Bradley (1994); Boot and Thakor (1997); Dow and Gorton (1997); Subrahmanyam and Titman (1999); and Fulghieri and Lukin (2001). Several recent papers in this literature are more closely related to the mechanism in our paper. Ozdenoren and Yuan (2007) show that the feedback effect from asset prices to the real value of a firm generates strategic complementarities. In their paper, however, the feedback effect is modeled exogenously and is not based on learning. Goldstein and Guembel (2008) do analyze learning by a decision maker, and show that this might lead to manipulation of the price by a single potentially informed trader. Hence, the manipulation equilibrium in their paper is not a result of strategic complementarities among heterogeneously informed traders. ${ }^{7}$ Dow, Goldstein, and Guembel (2008) show that the feedback effect generates complementarities in the decision to produce information, but not in the trading decision. ${ }^{8}$ Overall, the new insight in our paper - informational complementarities in trading due to the feedback effect - has not been explored in this literature, and thus with proper modeling can lead to a new contribution in the context of corporate finance. ${ }^{9}$

\footnotetext{
${ }^{7}$ See also Khanna and Sonti (2004), where manipulation happens as a result of the feedback effect. In their paper, feedback is exogenous and not based on learning.

${ }^{8}$ Complementarities in the decision to produce information also arise due to other reasons in several other papers. For example see, Froot, Scharfstein, and Stein (1992); Hirshleifer, Subrahmanyam, and Titman (1994); Bru and Vives (2002); and Veldkamp (2006a and 2006b).

${ }^{9}$ Our paper can be linked to contexts that are even beyond financial markets. For example, a typical problem in political economics involves a policymaker trying to learn from lobbying groups (e.g., in Battaglini and Benabou (2003)). The forces exposed in our paper, where agents coordinate on common pieces of
} 
The mechanism in our paper can also be linked to the vast herding literature that followed Scharfstein and Stein (1990). In their model, career-concerned managers make investment decisions sequentially. They tend to follow the decisions of their predecessors, wishing to convey to the public that their information is correlated with the information of others, and thus is likely to be of high quality. Our mechanism is different since it does not rely on career concerns, and because traders act simultaneously without observing what other traders do. Finally, in a concurrent and independent paper, Angeletos, Lorenzoni, and Pavan (2007) analyze how learning by Wall Street traders from aggregate investments of "Silicon Valley" firms can lead to informational complementarities. Our paper is different from theirs in the context of the study and in the modeling device. Hence, the two papers yield different results and implications.

The remainder of this paper is organized as follows. In Section 2, we present the model setup. Section 3 characterizes the equilibrium of the model. In Section 4, we describe the notion of informational complementarities that emerges in our model and how it leads to currency attacks. Section 5 analyzes the effectiveness of learning by the central bank from market outcomes, and shows how non-fundamental volatility emerges in exchange rates and policy making. In Section 6, we analyze the desirability of two policy tools in our model. Section 7 concludes.

\section{The Model Setup}

\section{$2.1 \quad$ Payoffs}

The players in our model are a central bank and a continuum of currency speculators. Initially, there is a currency peg in place. The central bank has to make a decision whether to maintain the currency peg or not. The value of maintaining the currency peg is characterized by a random state of the fundamental $\theta$. This fundamental may represent the terms of trade, the level of productivity in the economy, or the state of the banking system, as all these variables determine the prospects of the domestic currency and hence the desirability of maintaining the peg at the existing level. The fundamental $\theta$ can also represent political factors affecting the viability of the peg.

information due to informational complementarities may shed new light on the problems studied in this literature. 
The regime outcome is given by $\delta \in\{0,1\}$ where $\delta=1$ indicates that the central bank defends the status quo and $\delta=0$ indicates that the central bank abandons the status quo. The regime outcome $\delta$ is controlled by the central bank, whose payoff is given by

$$
U=\delta \theta
$$

Clearly, if the central bank was perfectly informed about the fundamental, the optimal decision would be to set $\delta=1$ if $\theta>0$ and set $\delta=0$ if $\theta<0$. In reality, the central bank is likely to be imperfectly informed, and our analysis focuses on the central bank's exchange rate policy in this case.

A continuum of speculators of measure one, indexed by $i$ and uniformly distributed over $[0,1]$, decide whether to short-sell the currency (i.e., attack the regime) or not. We assume that speculators are wealth constrained and can only short-sell up to one unit of the currency. The payoff of a speculator who does not attack is normalized to zero. The payoff from attacking the currency is $1-c$ if the status quo is abandoned (i.e., if the central bank sets $\delta=0)$ and $-c$ otherwise. Here, $c \in(0,1)$ is the opportunity cost of attacking. ${ }^{10}$

\subsection{Timing}

The central bank and the speculators play the following game. First, both the central bank and the speculators receive information regarding $\theta$. Then, the speculators independently and simultaneously decide whether to attack the currency or not. Finally, after observing the size of the aggregate attack from speculators, the central bank decides whether to maintain the status quo or not. Note that, unlike in the existing literature, the size of the speculative attack does not enter the central bank's payoff function in Equation (1). In our model, the effect of the speculative attack is due to the information revealed by the attack about the realization of the fundamental $\theta$.

\footnotetext{
${ }^{10}$ To simplify the algebra we follow some of the recent literature and assume that the payoff for speculators in case of devaluation is fixed. Alternatively, we could model the exchange rate determination by allowing the central bank to devalue the currency to the expected value of the fundamental, in which case the speculators' payoff would depend on the fundamental. This alternative setup would not change the basic forces of our model.
} 


\subsection{Information}

We assume that the central bank and the speculators have a common prior about $\theta$ which is an improper uniform over $\mathbb{R}$. The central bank receives a private signal $s_{b}=\theta+\sigma_{b} \epsilon_{b}$ about the fundamental, where $\epsilon_{b}$ is standard normally distributed (i.e., with mean of zero and standard deviation of one). We denote the p.d.f. and the c.d.f. of the standard normal distribution by $\phi$ and $\Phi$, respectively. We denote the precision of the central bank's signal by $\tau_{b}=1 / \sigma_{b}^{2}$. The central bank also observes the size of the attack $A$ from speculators. To reduce notational complexity, we use the variable $T \equiv \Phi^{-1}(A)$ instead of $A$ in analyzing the model. We assume that speculator $i \in[0,1]$ receives a signal $s_{i}=\theta+\sigma_{s} \epsilon_{i}$ about the fundamental, where $\epsilon_{i}$ is standard normally distributed. The precision of this signal is denoted by $\tau_{s}=1 / \sigma_{s}^{2}$. Notice that we are not assuming that speculators individually are better informed than the central bank. In fact, each speculator's information might be much noisier than the central bank's information.

Finally, we assume that speculators receive a common noisy signal about the fundamental, in addition to their private signals. We denote the common signal by $s_{p}=\theta+\sigma_{p} \epsilon_{p}$ where $\epsilon_{p}$ is standard normally distributed. The precision of this signal is denoted by $\tau_{p}=1 / \sigma_{p}^{2}$. We assume that this signal is not observed by the central bank. We will elaborate on the role of this assumption and different possible justifications in Section 4. Importantly, however, the key aspect of the information structure that is important for our results is that speculators share some correlated information that may facilitate coordination. Indeed, in Appendix B, we show that in an alternative setup where speculators receive correlated heterogenous signals instead of a common signal, the main results of the paper go through, except with complicated algebraic expressions. The payoff and information structure are common knowledge. All error terms $-\epsilon_{b}, \epsilon_{p}$, and $\epsilon_{i}$ - are independent of each other; the $\epsilon_{i}$ terms are also independent across investors.

\section{Equilibrium}

We now formally define an equilibrium in our model. Let $g\left(s_{i}, s_{p}\right)$ denote the action of a speculator given private signal $s_{i}$ and common signal $s_{p}, T\left(\theta, s_{p}\right)$ the size of the aggregate attack from speculators for given fundamental $\theta$ and common signal $s_{p}$, and $\delta\left(T, s_{b}\right)$ the action of the central bank as a function of the size of the attack and its signal. Furthermore, let $\nu\left(\theta \mid T, s_{b}\right)$ denote the posterior distribution of $\theta$ conditional on the central bank's infor- 
mation, and let $\mu\left(\theta \mid s_{i}, s_{p}\right)$ denote the posterior distribution of $\theta$ conditional on a speculator's information.

Definition 1 An equilibrium consists of a strategy for the central bank, $\delta\left(T, s_{b}\right)$, a symmetric strategy for the agents, $g\left(s_{i}, s_{p}\right)$, probability measures, $\nu\left(\cdot \mid T, s_{b}\right)$ and $\mu\left(\cdot \mid s_{i}, s_{p}\right)$, such that

$$
\begin{aligned}
& \delta\left(T, s_{b}\right) \in \operatorname{argmax}_{\delta \in\{0,1\}} \int_{-\infty}^{\infty} \delta \theta d \nu\left(\theta \mid T, s_{b}\right), \\
& g\left(s_{i}, s_{p}\right) \in \operatorname{argmax}_{a \in\{0,1\}} a \cdot\left[\int_{-\infty}^{\infty} \int_{-\infty}^{\infty} \mathbf{1}_{\left[\delta\left(T\left(\theta, s_{p}\right), \theta+\sigma_{b} \epsilon_{b}\right)=0\right]} d \mu\left(\theta \mid s_{i}, s_{p}\right) d \Phi\left(\epsilon_{b}\right)-c\right], \\
& T\left(\theta, s_{p}\right)=\Phi^{-1}\left(\int_{-\infty}^{\infty} g\left(\theta+\sigma_{s} \epsilon, s_{p}\right) \phi(\epsilon) d \epsilon\right), \\
& \nu\left(\theta \mid T, s_{b}\right) \text { is obtained using Bayes' rule for any } T \text { and } s_{b}, \\
& \mu\left(\theta \mid s_{i}, s_{p}\right) \text { is obtained using Bayes' rule for any } s_{i} \text { and } s_{p} .
\end{aligned}
$$

Our focus will be on linear threshold equilibria. These are equilibria where speculators attack the currency if and only if their private signal $s_{i}$ is below a threshold $\hat{s}\left(s_{p}\right)$, which is a linear function of the common signal $s_{p}$. In addition, the central bank abandons the regime if and only if the aggregate size of the attack $T$ is above a threshold $\hat{T}\left(s_{b}\right)$, which is a function of its private signal $s_{b}$. The next proposition shows that there is a unique such equilibrium and characterizes it.

Proposition 1 There is a unique linear threshold equilibrium where the speculators' threshold strategy is:

$$
g\left(s_{i}, s_{p}\right)=\left\{\begin{array}{lll}
1 & \text { if } \quad s_{i} \leq \hat{s}\left(s_{p}\right) \\
0 & \text { if } \quad s_{i}>\hat{s}\left(s_{p}\right)
\end{array},\right.
$$

and the central bank's strategy is

$$
\delta\left(T, s_{b}\right)=\left\{\begin{array}{lll}
1 & \text { if } \quad T \leq \hat{T}\left(s_{b}\right) \\
0 & \text { if } \quad T>\hat{T}\left(s_{b}\right)
\end{array} .\right.
$$

Here,

$$
\hat{s}\left(s_{p}\right)=\hat{s}(0)-k s_{p},
$$

where $k>0$ is the unique real root of the cubic equation:

$$
-\frac{\tau_{b} \tau_{s}}{\tau_{p}} k^{3}+\left(\tau_{b}+\tau_{p}\right) k^{2}+2 \tau_{p} k+\tau_{p}=0
$$


and $\hat{s}(0)$ satisfies

$$
c=\Phi\left(\frac{-\left((1+k) \frac{\tau_{b}}{\tau_{T}}+1\right) \frac{\tau_{s}}{\tau_{s}+\tau_{p}} \hat{s}(0)}{\sqrt{\left((1+k) \frac{\tau_{b}}{\tau_{T}}+1\right)^{2} \frac{1}{\tau_{s}+\tau_{p}}+(1+k)^{2} \frac{\tau_{b}}{\tau_{T}^{2}}}}\right),
$$

and

$$
\hat{T}\left(s_{b}\right)=\frac{1}{\sigma_{s}}\left[\hat{s}(0)+(1+k) \frac{\tau_{b}}{\tau_{T}} s_{b}\right],
$$

where

$$
\tau_{T}=\tau_{p}\left(1+\frac{1}{k}\right)^{2}
$$

is the precision of the attack as a signal of the fundamental.

\section{PROOF OF PROPOSITION 1:}

Suppose an agent attacks if and only if $s_{i}+k s_{p} \leq \hat{s}(0)$ where $k>0$. The size of the attack from speculators given $\theta$ and $s_{p}$ is $A\left(\theta, s_{p}\right)=\Phi\left(\frac{\hat{s}(0)-k s_{p}-\theta}{\sigma_{s}}\right)$. The central bank observes $T\left(\theta, s_{p}\right)=\Phi^{-1}(A)$, or equivalently, it observes

$$
T=\frac{\hat{s}(0)-k s_{p}-\theta}{\sigma_{s}},
$$

which can be rewritten as

$$
\frac{\hat{s}(0)-\sigma_{s} T}{1+k}=\theta+\frac{k \sigma_{p}}{1+k} \epsilon_{p}
$$

Thus, the precision of the attack as a signal of the fundamental is

$$
\tau_{T}=\frac{\tau_{p}(1+k)^{2}}{k^{2}}
$$

and

$$
E\left[\theta \mid T, s_{b}\right]=\frac{\tau_{T}}{\tau_{T}+\tau_{b}}\left(\frac{\hat{s}(0)-\sigma_{s} T}{1+k}\right)+\frac{\tau_{b}}{\tau_{T}+\tau_{b}} s_{b} .
$$

This implies the status quo is abandoned if and only if

$$
T \geq \frac{\hat{s}(0)}{\sigma_{s}}+\frac{1+k}{\sigma_{s}} \frac{\tau_{b}}{\tau_{T}} s_{b}=\hat{T}\left(s_{b}\right)
$$

which is Equation (4). For a speculator, $\theta$ is distributed with mean $\frac{\tau_{s}}{\tau_{s}+\tau_{p}} s_{i}+\frac{\tau_{p}}{\tau_{s}+\tau_{p}} s_{p}$ and precision $\tau_{s}+\tau_{p}$. The posterior belief of the regime change for a speculator with signal $s_{i}$ 
and $s_{p}$ is expressed as follows:

$$
\begin{aligned}
& \operatorname{Pr}\left(T \geq \frac{\hat{s}(0)}{\sigma_{s}}+\frac{1+k}{\sigma_{s}} \frac{\tau_{b}}{\tau_{T}} s_{b} \mid s_{i}, s_{p}\right) \\
= & \operatorname{Pr}\left(\left((1+k) \frac{\tau_{b}}{\tau_{T}}+1\right) \theta+(1+k) \frac{\tau_{b}}{\tau_{T}} \sigma_{b} \epsilon_{b} \leq-k s_{p} \mid s_{i}, s_{p}\right) \\
= & \Phi\left(\frac{-\left((1+k) \frac{\tau_{b}}{\tau_{T}}+1\right) \frac{\tau_{s}}{\tau_{s}+\tau_{p}} s_{i}-\left(\left((1+k) \frac{\tau_{b}}{\tau_{T}}+1\right) \frac{\tau_{p}}{\tau_{s}+\tau_{p}}+k\right) s_{p}}{\sqrt{\left((1+k) \frac{\tau_{b}}{\tau_{T}}+1\right)^{2} \frac{1}{\tau_{s}+\tau_{p}}+\left((1+k) \frac{\tau_{b}}{\tau_{T}} \sigma_{b}\right)^{2}}}\right)
\end{aligned}
$$

In equilibrium the threshold strategy must satisfy the following equation for all $s_{p}$ :

$$
c=\Phi\left(\frac{-\left((1+k) \frac{\tau_{b}}{\tau_{T}}+1\right) \frac{\tau_{s}}{\tau_{s}+\tau_{p}} \hat{s}(0)-\left(\left((1+k) \frac{\tau_{b}}{\tau_{T}}+1\right)\left(\frac{\tau_{p}}{\tau_{s}+\tau_{p}}-\frac{\tau_{s}}{\tau_{s}+\tau_{p}} k\right)+k\right) s_{p}}{\sqrt{\left((1+k) \frac{\tau_{b}}{\tau_{T}}+1\right)^{2} \frac{1}{\tau_{s}+\tau_{p}}+\left((1+k) \frac{\tau_{b}}{\tau_{T}} \sigma_{b}\right)^{2}}}\right) .
$$

Thus, for a linear equilibrium to exist the coefficient of $s_{p}$ must be zero. In other words, $k$ must satisfy:

$$
-k \frac{\tau_{s}}{\tau_{s}+\tau_{p}}+\frac{\tau_{p}}{\tau_{s}+\tau_{p}}+\frac{k}{\left(1+(1+k) \frac{\tau_{b}}{\tau_{T}}\right)}=0 .
$$

Substituting for $\tau_{T}$ and rearranging the above equation we obtain:

$$
-\frac{\tau_{b} \tau_{s}}{\tau_{p}} k^{3}+\left(\tau_{b}+\tau_{p}\right) k^{2}+2 \tau_{p} k+\tau_{p}=0
$$

The above cubic equation has a single real root since the discriminant $\Delta$ given below is strictly positive:

$$
\Delta=\tau_{b}\left(4 \tau_{p}^{3}+8 \tau_{p}^{2} \tau_{b}+4 \tau_{p}^{2} \tau_{s}+4 \tau_{p} \tau_{b}^{2}+36 \tau_{p} \tau_{b} \tau_{s}+27 \tau_{b} \tau_{s}^{2}\right)>0 .
$$

To complete the construction of a linear equilibrium, we need to show that this root is strictly positive. Note that the left side of Equation (7) goes to $\infty$ as $k$ goes to $-\infty$ and $-\infty$ as $k$ goes to $\infty$. Moreover, it is positive at $k=0$. Since the equation has a single real root, it must cross zero at some $k>0$.

In equilibrium, the optimal strategy for a speculator who receives a signal $s_{i}$ is to attack if and only if $s_{i}$ falls below a threshold value, $\hat{s}\left(s_{p}\right)$, which is decreasing in the common noisy signal $s_{p}$. That is, when the common signal indicates a sound fundamental, speculators attack only if their private signals are very pessimistic. The weight $k$ that the speculator puts on the common signal is derived endogenously. For the central bank, the attack provides 
an additional signal about the fundamental. This signal has precision $\tau_{T}$ which is decreasing in the weight $k$ that speculators put on the common signal. The optimal strategy for the central bank is to abandon the exchange rate regime if and only if the observed signal of aggregate attack, $T$, is greater than or equal to the threshold, $\hat{T}\left(s_{b}\right)$, which is increasing in the central bank's private signal $s_{b}$.

\section{Informational Complementarities and Currency At- tacks}

An important element of our equilibrium is $k$ - the weight that speculators put on the common signal $s_{p}$ in their decision whether to participate in a speculative attack. At a basic level, speculators put a positive weight on $s_{p}$ because it provides additional information about the realization of $\theta$, and thus on the probability that the central bank will abandon the regime. Because speculators know that the central bank is going to use the information conveyed by the size of the attack in its policy decision, however, they end up putting too much weight on $s_{p}$.

To see this, let us compare the weight $k$ that speculators put on the common signal $s_{p}$ (implicitly defined in Equation (3)) with a benchmark level $k_{B M}$ that would be obtained if the central bank did not use the size of the attack to infer information about the fundamental $\theta$. The next proposition shows that $k$ - defined in Equation $(3)$-is indeed greater than $k_{B M}$.

Proposition 2 The weight $k$ put by speculators on $s_{p}$ in the unique linear threshold equilibrium characterized by Proposition 1 is greater than the weight $k_{B M}$ that would be put on $s_{p}$ in a game where the central bank does not attempt to get information about $\theta$ from the size of the attack.

\section{PROOF OF PROPOSITION 2:}

We start by computing $k_{B M}$. When the central bank does not update its belief about $\theta$ based on the size of the attack, it will abandon the regime if and only if its private signal $s_{b}=\theta+\sigma_{b} \epsilon_{b}$ is negative. Then, based on the logic used in the proof of Proposition 1 , speculators will attack the currency if and only if $\operatorname{Pr}\left(\theta+\sigma_{b} \epsilon_{b}<0 \mid s_{i}, s_{p}\right)>c$.

In a linear threshold equilibrium, speculators attack if and only if their private signal $s_{i}$ is below the threshold $\hat{s}_{B M}\left(s_{p}\right)=\hat{s}_{B M}(0)-k_{B M} s_{p}$. Thus, the speculator who observes the 
threshold signal has to be indifferent between attacking and not attacking:

$$
\operatorname{Pr}\left(\theta+\sigma_{b} \epsilon_{b}<0 \mid \hat{s}_{B M}\left(s_{p}\right), s_{p}\right)=c .
$$

Since $\theta$ and $\epsilon_{b}$ are independently normally distributed, this implies that:

$$
\Phi\left(\frac{-\frac{\tau_{s}}{\tau_{s}+\tau_{p}} \hat{s}_{B M}\left(s_{p}\right)-\frac{\tau_{p}}{\tau_{s}+\tau_{p}} s_{p}}{\sqrt{\frac{1}{\tau_{s}+\tau_{p}}+\sigma_{b}^{2}}}\right)=c .
$$

Plugging in $\hat{s}_{B M}\left(s_{p}\right)=\hat{s}_{B M}(0)-k_{B M} s_{p}$, we get:

$$
\Phi\left(\frac{-\frac{\tau_{s}}{\tau_{s}+\tau_{p}} \hat{s}_{B M}(0)-\left(\frac{\tau_{p}}{\tau_{s}+\tau_{p}}-\frac{\tau_{s}}{\tau_{s}+\tau_{p}} k_{B M}\right) s_{p}}{\sqrt{\frac{1}{\tau_{s}+\tau_{p}}+\sigma_{b}^{2}}}\right)=c .
$$

Since this equation has to hold for every $s_{p}$, we get:

$$
k_{B M}=\frac{\tau_{p}}{\tau_{s}}
$$

Now, we show that $k$ (defined in Equation (3)) is greater than $k_{B M}$. To do this, we need to evaluate the left side of Equation (3) at $k=k_{B M}=\frac{\tau_{p}}{\tau_{s}}$. This yields:

$$
\begin{aligned}
& -\frac{\tau_{b} \tau_{p}^{2}}{\tau_{s}^{2}}+\left(\tau_{b}+\tau_{p}\right) \frac{\tau_{p}^{2}}{\tau_{s}^{2}}+2 \frac{\tau_{p}^{2}}{\tau_{s}}+\tau_{p} \\
= & \frac{\tau_{p}}{\tau_{s}^{2}}\left(\tau_{p}^{2}+2 \tau_{p} \tau_{s}+\tau_{s}^{2}\right)>0 .
\end{aligned}
$$

Hence, $k>k_{B M}$.

Intuitively, the fact that the central bank learns from the size of the attack and uses the information in its policy decision generates strategic complementarities among speculators. Seeing a large attack, the central bank is more likely to believe that $\theta$ is low and to abandon the regime. Thus, when a speculator thinks that other speculators are more likely to attack, his incentive to attack also increases. This pushes speculators to put a larger weight on $s_{p}$, which provides information not only about $\theta$ but also about the information that other speculators have on $\theta$ and hence about what other speculators are going to do. Unlike in Morris and Shin (1998, 2002), strategic complementarities here emerge endogenously as a result of learning, and are not directly imposed on the payoff functions. We thus distinguish the informational complementarities in our setting from the direct complementarities in the previous literature.

Informational complementarities lead to coordination motives among speculators in our model. These coordination motives generate large currency attacks that are not justified by 
fundamentals. Essentially, when $s_{p}$ is low due to unusual common noise, a large currency attack will be formed despite the fundamentals being reasonably high. In the next sections, we develop the main implications of these complementarities in our framework, concerning the effectiveness of learning from the market, non-fundamental volatility, time-consistency, and transparency in exchange-rate policy.

Before turning to these results, we should clarify the role of the common signal $s_{p}$. The common signal is essentially the device via which informational complementarities manifest themselves in our model. Speculators coordinate on attacking more (less) aggressively when the common signal they observe is low (high). Since the central bank does not observe the common signal, it does not know if a large attack is due to coordination or information, and so it can be "fooled" to take the wrong action. The assumption that the central bank does not observe a signal that is commonly observed by all speculators may be justified by the fact that in currency attacks speculators usually come from a foreign market, and thus may have information that is not available to the central bank.

More importantly, we should stress that our results do not depend on this assumption. Our model only requires that the speculators will be able to coordinate their actions through some commonality of their information, which the central bank cannot fully tease out. In fact, our results go through in alternative information structures, where this commonality is not so extreme. Here are a few examples. First, our results can be preserved in a symmetric information environment where both the central bank and the speculators observe the common signal with some given probability less than one. In this case, since there is a continuum of speculators, a fixed proportion of the speculators always observe the common signal. Moreover, the speculators know that with a positive probability the central bank has missed this signal; hence, there is still room for the coordination motive to play a role, and the basic insights of our analysis continue to hold. Second, our results go through in an information environment where speculators observe correlated private signals, instead of a common signal. We fully develop such a framework in Appendix B. In this framework, speculators can still coordinate their actions by putting excess weight on their correlated signals, and the central bank cannot filter it out completely since the common component of these correlated signals is observed with noise by all agents in the economy. Third, in Appendix C, we show that a very similar result holds even when the speculators receive a common signal which is not fundamental related. ${ }^{11}$ That assumption is empirically plausible

\footnotetext{
${ }^{11}$ In that case, we assume that the central bank observes the size of the attack with noise and the speculators
} 
since speculators may share some common information regarding random shocks to the institutional environment or to the workings of the currency market, which are not known to the central bank (for example, traders may know other traders personally, and thus expect changes in their appetite for risk). ${ }^{12}$

We close this section by analyzing the determinants of $k$ in equilibrium.

Proposition 3 The equilibrium value of $k$ decreases in $\tau_{b}$ and $\tau_{s}$, and increases in $\tau_{p}$.

\section{PROOF OF PROPOSITION 3}

By taking the total derivative of Equation (7) with respect to $\tau_{b}$ we obtain:

$$
\frac{\partial k}{\partial \tau_{b}}=\frac{\frac{\tau_{s}}{\tau_{p}} k^{3}-k^{2}}{-3 \frac{\tau_{b} \tau_{s}}{\tau_{p}} k^{2}+2\left(\tau_{b}+\tau_{p}\right) k+2 \tau_{p}}
$$

To see the sign of the denominator of Equation (8) in equilibrium, we use the equilibrium condition given by Equation (7) to obtain:

$$
-3 \frac{\tau_{b} \tau_{s}}{\tau_{p}} k^{2}+2\left(\tau_{b}+\tau_{p}\right) k+2 \tau_{p}=\frac{-\left(\tau_{b}+\tau_{p}\right) k^{2}-4 \tau_{p} k-3 \tau_{p}}{k}<0 .
$$

Similarly, to see the sign of the numerator of Equation (8) in equilibrium, we once more use Equation (7) to obtain:

$$
\frac{\tau_{s}}{\tau_{p}} k^{3}-k^{2}=\frac{\tau_{p} k^{2}+2 \tau_{p} k+\tau_{p}}{\tau_{b}}>0 .
$$

Thus, $\frac{\partial k}{\partial \tau_{b}}<0$. Similarly,

$$
\begin{aligned}
\frac{\partial k}{\partial \tau_{p}} & =\frac{\frac{\tau_{b} \tau_{s}}{\tau_{p}^{2}} k^{3}+k^{2}+2 k+1}{3 \frac{\tau_{b} \tau_{s}}{\tau_{p}} k^{2}-2\left(\tau_{b}+\tau_{p}\right) k-2 \tau_{p}}>0, \\
\frac{\partial k}{\partial \tau_{s}} & =\frac{\frac{\tau_{b}}{\tau_{p}} k^{3}}{-3 \frac{\tau_{b} \tau_{s}}{\tau_{p}} k^{2}+2\left(\tau_{b}+\tau_{p}\right) k+2 \tau_{p}}<0 .
\end{aligned}
$$

are able to observe the noise component of the size of the attack.

${ }^{12}$ Related to this, one may ask whether the central bank can construct a mechanism to reveal the common signal by directly contacting speculators. While addressing this point formally is beyond the scope of our paper, we wish to point out that our results hold as long as speculators hold correlated private signals as shown in Appendix B. The common element in these correlated signals is not known to any agent in the economy so it is impossible for the central bank to observe it perfectly by contacting some speculators directly. Further, it is difficult to implement such mechanism in the real world. A key feature of financial markets is the anonymity of most speculators. This implies that it is difficult for the central bank to identify all speculators who are active in the market at a particular point in time. 
In other words, if the central bank's or the speculators' private information is less precise, or the common signal is more precise, speculators coordinate better in equilibrium so that the weight they put on the common signal increases. Intuitively, if the central bank holds a precise signal, it relies less on the information revealed in the aggregate actions of the speculators. In equilibrium, this forces the speculators to reduce the weight they put on the common signal and to reveal more information to the central bank. If each speculator holds a very sharp private signal about the fundamental, each bases the decision to attack mostly on the private signal rather than the noisy common signal, and hence there is less incentive to coordinate. Finally, the incentive to coordinate is largest when the common signal is very precise. In this case, the speculators put a larger weight on the common signal and the central bank cannot ignore the information revealed in the aggregate attack.

\section{The Effectiveness of Learning from the Market and Non-Fundamental Volatility}

We now analyze how informational complementarities impact the effectiveness of learning from the market. We do this by looking at the probability of policy mistakes. The central bank makes a policy mistake in our model when it abandons (maintains) the status quo given that $\theta>0(\theta<0)$. The following proposition characterizes the probability of making a policy mistake and studies its properties.

Proposition 4 The ex ante probability of abandoning the status quo for a given $\theta$ is

$$
\Phi\left(-\sqrt{\tau_{b}+\tau_{T}} \theta\right)
$$

Hence, when $\theta>0$, the probability of making a policy mistake is $\Phi\left(-\sqrt{\tau_{b}+\tau_{T}} \theta\right)$, while when $\theta<0$, it is $1-\Phi\left(-\sqrt{\tau_{b}+\tau_{T}} \theta\right)$.

\section{PROOF OF PROPOSITION 4:}

The ex ante probability of abandoning the status quo given $\theta$ is:

$$
\operatorname{Pr}\left(T \geq \frac{\hat{s}(0)}{\sigma_{s}}+\frac{1+k}{\sigma_{s}} \frac{\tau_{b}}{\tau_{T}} s_{b} \mid \theta\right)=\operatorname{Pr}\left(\epsilon_{b}+\frac{1}{\sigma_{b}} \frac{\sqrt{\tau_{T}}}{\tau_{b}} \epsilon_{p} \leq-\frac{1}{\sigma_{b}}\left(1+\frac{\tau_{T}}{\tau_{b}}\right) \theta \mid \theta\right)
$$

where the equality follows by plugging in for $T, s_{p}, s_{b}$ and rearranging Equation (6). The term $\epsilon_{b}+\frac{1}{\sigma_{b}} \frac{\sqrt{\tau_{T}}}{\tau_{b}} \epsilon_{p}$ is the weighted sum of two independent normal random variables, so this term itself is normal with mean 0 and variance $1+\frac{\tau_{T}}{\tau_{b}}$. Thus the ex-ante probability of 
abandoning the status quo for a given $\theta$ is given by:

$$
\operatorname{Pr}\left(\epsilon_{b}+\frac{1}{\sigma_{b}} \frac{\sqrt{\tau_{T}}}{\tau_{b}} \epsilon_{p} \leq-\frac{1}{\sigma_{b}}\left(1+\frac{\tau_{T}}{\tau_{b}}\right) \theta \mid \theta\right)=\Phi\left(-\sqrt{\tau_{b}+\tau_{T}} \theta\right) .
$$

Intuitively, the probability of making a policy mistake decreases in the precision of the two pieces of information that the central bank has: the precision of the information conveyed by the size of the attack $\tau_{T}$ and the precision of the central bank's private information $\tau_{b}$. Since $\tau_{T}$ is decreasing in the weight $k$ that speculators put on their common signal (see (5)), $k$, which is partly a result of informational complementarities, has a positive effect on the probability of a policy mistake.

This suggests that learning from the market has a self-defeating aspect. In Proposition 2, we showed that by learning from the market, the central bank gives rise to informational complementarities that increase the weight $k$ that speculators put on the common signal. Now we see that $k$ increases the probability of policy mistakes. Hence, when using the information from the market, the central bank reduces the quality of this information. It should be noted that informational complementarities are not the only source of policy mistakes in the model. As long as the precision of the common signal is non-zero, speculators will put a positive weight on this signal (see the definition of $k_{B M}$ above), and so the noise in the common signal will be reflected in the size of the attack and generate policy mistakes. Yet, the presence of complementarities - generated by the central bank's learning - amplifies the weight speculators put on the common signal, and increases the tendency for policy mistakes.

As a benchmark, it is interesting to consider what would happen in a model where the common signal played no role (i.e., when $\tau_{p}=0$.) As the next proposition shows, in this case, the speculators can no longer coordinate on the common signal, i.e., $k \rightarrow 0$. Then, the attack becomes fully revealing of the fundamental, as the noise terms of the idiosyncratic signals cancel out with each other, and the central bank does not make policy mistakes. ${ }^{13}$

Proposition 5 In the limit as $\tau_{p}$ approaches $0, \hat{s}\left(s_{p}\right)=\hat{s}(0)=\sigma_{s} \Phi^{-1}(1-c), \hat{T}\left(s_{b}\right)=$ $\Phi^{-1}(1-c)$, and the attack becomes fully revealing of $\theta$.

\footnotetext{
${ }^{13}$ In Appendix D, we study the other limit where the common signal becomes infinitely precise and hence the speculators commonly know the fundamental. We show that in this limit the linear equilibrium disappears, and there are multiple equilibria. In these equilibria, speculators coordinate their actions perfectly by either all of them or none of them attacking for a given value of the fundamental.
} 


\section{PROOF OF PROPOSITION 5:}

We start with the following lemma:

Lemma 1 (i) $\lim _{\tau_{p} \rightarrow 0} k\left(\tau_{p}\right)=0$, (ii) $\lim _{\tau_{p} \rightarrow 0} k\left(\tau_{p}\right) / \tau_{p}=\infty$, and (iii) $\lim _{\tau_{p} \rightarrow 0} k\left(\tau_{p}\right)^{2} / \tau_{p}=0$.

PROOF OF LEMMA 1 To see that $k\left(\tau_{p}\right)$ approaches zero as $\tau_{p}$ approaches zero, first recall that $\partial k / \partial \tau_{p}$ is positive. Thus as $\tau_{p}$ approaches zero, $k\left(\tau_{p}\right)$ has a limit that is less than infinity. Suppose that this limit is strictly positive. Then it is easy to see that

$$
-\frac{\tau_{b} \tau_{s}}{\tau_{p}} k^{3}+\left(\tau_{b}+\tau_{p}\right) k^{2}+2 \tau_{p} k+\tau_{p}<0
$$

for $\tau_{p}$ small enough which is a contradiction, establishing that $\lim _{\tau_{p} \rightarrow 0} k\left(\tau_{p}\right)=0$.

By letting $z=\frac{k}{\tau_{p}}$, Equation (3) can be rewritten as

$$
-\tau_{b} \tau_{s} z^{3}+\left(\tau_{b}+\tau_{p}\right) z^{2}+2 z+\frac{1}{\tau_{p}}=0
$$

From the above equation we see that as $\tau_{p}$ approaches zero, $z=\frac{k}{\tau_{p}}$ approaches $\infty$. Moreover, from Equation (3),

$$
\left(\frac{k^{2}}{\tau_{p}}\right)=\frac{(2 k+1)}{\tau_{b} \tau_{s} \frac{k}{\tau_{p}}-\left(\tau_{b}+\tau_{p}\right)}
$$

and the right side approaches zero as $\tau_{p}$ approaches zero, establishing $\lim _{\tau_{p} \rightarrow 0} k\left(\tau_{p}\right)^{2} / \tau_{p}=0$.

Using Lemma 1, we see that as $\tau_{p}$ approaches zero,

$$
c=\Phi\left(\frac{-\left(\frac{\tau_{b}}{(1+k)} \frac{k^{2}}{\tau_{p}}+1\right) \frac{\tau_{s}}{\tau_{s}+\tau_{p}} \hat{s}(0)}{\sqrt{\left(\frac{\tau_{b}}{(1+k)} \frac{k^{2}}{\tau_{p}}+1\right)^{2} \frac{1}{\tau_{s}+\tau_{p}}+\tau_{b} \frac{k^{2}}{\tau_{p}}}}\right) \rightarrow 1-\Phi\left(\sqrt{\tau_{s}} \hat{s}(0)\right)
$$

and

$$
\hat{T}\left(s_{b}\right)=\frac{1}{\sigma_{s}}\left[\hat{s}(0)+\frac{k^{2} \tau_{b}}{\tau_{p}(1+k)} s_{b}\right] \rightarrow \frac{1}{\sigma_{s}} \hat{s}(0)=\Phi^{-1}(1-c) .
$$

To see that the limiting equilibrium is fully revealing, note that a speculator attacks if and only if $s \leq \hat{s}(0)$. The size of the attack from speculators is $A(\theta)=\Phi\left(\frac{\hat{s}(0)-\theta}{\sigma_{s}}\right)$, and the central bank observes $T(\theta)=\Phi^{-1}(A)$ or $T=(\hat{s}(0)-\theta) / \sigma_{s}$. Hence, in equilibrium, the central bank infers $\theta$ perfectly which is given by:

$$
\theta=\hat{s}-\sigma_{s} T .
$$

Taken together, the two propositions above generate a puzzling result. Even though speculators are better informed about the fundamental when they observe a common signal 
in addition to their private signals, the central bank, paradoxically, becomes less informed when this signal is introduced. This suggests that adding a source of information reduces the effectiveness of the learning process of the central bank. We now explore this aspect of the model more fully by analyzing the effect that the precision of the various signals in our model has on the effectiveness of learning from the market. The following proposition provides the main result.

Proposition 6 Conditional on $\theta$, the ex-ante probability of making a policy mistake decreases in $\tau_{b}$ and $\tau_{s}$, increases in $\tau_{p}$ if $0<\tau_{p}<\tau_{b} \frac{\sqrt{1+16 \frac{\tau_{s}}{\tau_{b}}}-1}{8}$ and decreases in $\tau_{p}$ if $\tau_{p}>$ $\tau_{b} \frac{\sqrt{1+16 \frac{\tau_{s}}{\tau_{b}}}-1}{8}$.

PROOF OF PROPOSITION 6: See Appendix A.

The results regarding $\tau_{s}$ and $\tau_{b}$ are straightforward. Improving the precision of the speculators' private signals or of the central bank's private signal generates a decrease in the probability that the central bank will make a policy mistake. But the effect of increasing the precision $\tau_{p}$ of the common signal can go in both directions. On the one hand, increasing the precision of the common signal implies that speculators have access to more precise information, which can be revealed to the central bank via the trading process. On the other hand, increasing the precision of the common signal implies that speculators will rely more on the common signal. That is, the ability of speculators to coordinate on the common signal and convey a misleading message to the central bank improves. Our result shows that the first effect dominates when $\tau_{p}$ is above a certain cutoff and the second effect dominates when $\tau_{p}$ is below the cutoff. Hence, the probability of a policy mistake is maximized at an intermediate level of $\tau_{p}$.

Another way to view our results is that informational complementarities lead to nonfundamental volatility in the exchange rate regime. Due to informational complementarities, the noise in the common signal gets to have a significant impact on speculators' tradings and leads to many instances where the exchange rate regime is abandoned without the fundamentals justifying it. In that, our model rationalizes the empirical results documented by Eichengreen, Rose, and Wyplosz (1995), showing that there are excessive transitions between exchange rate regimes and that these transitions cannot be explained by fundamentals. Our model also suggests that such transitions are more likely to happen when speculators are exposed to some common information of moderate precision. Policy mistakes may also lead to a rebound in the exchange rate after a speculative attack leads to devaluation. Such 
rebounds are commonly observed, and led to a large theoretical literature attributing them to multiple equilibria (see Flood and Garber (1984), Obstfeld (1986), and others). Our paper suggests a different mechanism behind such frequent rebounds which is linked to the structure of information in the foreign exchange market. For example, devaluations that follow speculative attacks are more likely to be reversed in an environment where the private source of information available to speculators is imprecise and the common information is moderately precise.

An empirical exploration of our theoretical results may use data on correlations in currency speculators' tradings over time to gauge the degree to which they rely on private vs. common information. In the market microstructure literature, such data is used in constructing the PIN measure which assesses the level of informativeness in the trading process (see, for example, Easley, Hvidkjaer, and O'Hara (2002)). This measure has also been used to investigate whether information in financial markets has an effect on real decisions (Chen, Goldstein, and Jiang (2007)).

We conclude this section by pointing to another interesting property of speculative attacks in our model, which stands in stark difference to existing models of currency attacks. This is summarized in the next corollary.

Corollary 1 The level of the opportunity cost, c (as well as the wealth level of speculators) affects the size of the attack but does not affect the information content of the attack and, hence, does not affect the probability of devaluation occurring for a given $\theta$.

The reason for this result is that the central bank does not care about the size of the attack per se when making a policy decision. A large attack will induce the central bank to abandon the exchange rate regime only if it provides information that the fundamentals are low. Hence, while a decrease in the cost of attacking the regime will increase the tendency of speculators to attack, this will be filtered out by the central bank, and will not change the overall tendency to abandon the regime. The implication is that when we compare across markets, some speculative attacks will be defended, while others that are weaker (but provide more information) will not.

\section{$6 \quad$ Policy Implications}

We now analyze the effect of two policy tools on the effectiveness of learning from the market. 


\subsection{Commitment}

Knowing that the central bank uses the information in the size of the attack, speculators put a too large weight on the common signal. This, in turn, reduces the informativeness of the size of the attack and harms the effectiveness of the learning process. The first policy tool that we explore is commitment by the central bank to reduce the weight it puts on the information in the size of the attack below what is ex-post optimal. There are two effects to consider in evaluating this policy tool. First, there is obviously a cost in deviating from the ex-post optimal decision. When doing that, the central bank does not fully use all available information, which causes a reduction in its ability to achieve its objectives. Second, by committing to put a low weight on the size of the attack, the central bank affects the exante trading motives of speculators. In particular, under such policy, speculators will find it less valuable to coordinate on the common signal, and this will improve the quality of the information conveyed by the attack. The next proposition analyzes the overall desirability of such a commitment policy.

Proposition 7 Suppose that speculators follow linear strategies, then the central bank can always increase its expected payoff by committing to put a slightly lower (higher) weight on the information in the attack (on its private signal) than is ex-post optimal.

\section{PROOF OF PROPOSITION 7: See Appendix A.}

The proposition says that it is always optimal for the central bank to commit to a slight deviation from the ex-post optimal weights, and in particular to increase the weight given to its private signal and decrease the weight given to the information in the size of the attack. The reason is that the cost of a small deviation from the ex-post optimal weight approaches 0 . On the other hand, the benefit from the increased informativeness of the attack is always strictly positive. As a result, slight deviations from ex-post optimal weights are always desirable. Of course, large deviations are not desirable. For example, it is never optimal for the central bank to completely ignore the attack as a signal for the fundamentals. This can be seen by comparing the probability of devaluation for a given $\theta$ when the central bank ignores the attack which is given by $\Phi\left(-\sqrt{\tau_{b}} \theta\right)$ and the probability of devaluation when the central bank learns from the attack which is given by $\Phi\left(-\sqrt{\tau_{b}+\tau_{T}} \theta\right)$. The latter probability is smaller when $\theta>0$ and larger when $\theta<0$.

Proposition 7 exposes a problem of time inconsistency in the central bank's policy. While the central bank wants to commit ex ante to put a lower weight on the information coming 
from speculative attacks, once the attack is realized ex post, the central bank would always be tempted to pay more attention to it. Hence, the feasibility of the policy tool analyzed here depends critically on the central bank's commitment power. As a result, overconfident central bankers can achieve better outcomes. Being overconfident about the precision of their own information, central bankers effectively provide a guarantee that they will not much weight on the information in speculative attacks and encourage speculators to put less weight on the common signal in their trading decisions. ${ }^{14}$

\subsection{Transparency}

Next, we discuss the issue of central bank transparency, in other words, whether and how clearly the central bank should communicate its information to the public. The issue of central bank transparency is receiving a lot of attention in research and policy circles. The positive aspects of transparency are often emphasized. In a recent paper, Morris and Shin (2005) demonstrate a cost associated with transparency. Building on the insight in Morris and Shin (2002), they show that in the presence of direct complementarities among market participants, transparency can be bad because it provides a public signal, and thus reduces the extent to which speculators use their private information. This, in turn, reduces the ability of the central bank to infer new information from the market.

We analyze the effect of transparency in our framework, where there are no direct complementarities among speculators, but rather speculators care about each other's strategies because they know that their collective action reveals information that affects the central bank's policy decision. We discover a new negative effect of transparency. When the central bank becomes more transparent, it reveals information about the course of action that it is likely to take in the future. Knowing this, the speculators can better coordinate on conveying a misleading signal. To see this, consider the extreme case where the central bank perfectly reveals its information, and suppose that speculators know that solely on the basis of this information, the central bank would devalue. In this case, it is an equilibrium for all the speculators to attack. Since the attack reveals no information the central bank will indeed devalue, and completely miss the opportunity to learn from the market to shape its policy decision. The next proposition analyzes the more interesting case where the central

\footnotetext{
${ }^{14}$ This result is similar in intuition to that obtained in Bolton, Brunnermeier and Veldkamp (2007). They show that over-confidence is a valuable leadership attribute since it helps leaders to stick to their prior belief when constantly learning about the optimal action in a changing environment.
} 
bank releases its information with some noise. It shows that as long as this information is commonly observed by the speculators, the central bank is more likely to make a policy mistake.

Proposition 8 Suppose that the central bank releases a public signal $s_{a}=s_{b}+\sigma_{a} \epsilon_{a}$ where $\epsilon_{a} \sim N(0,1)$. The weight $k_{t r}$ put by speculators on $s_{p}$ in the unique linear threshold equilibrium is bigger than that characterized by Proposition 1.

PROOF OF PROPOSITION 8: See Appendix A.

The proposition indicates that the central bank may inadvertently strengthen the coordination incentive by releasing more information that becomes common to the speculators. In fact, the more precise such information is, the stronger the coordination among speculators (i.e., the higher $k$ ). As discussed above, this result is due to the fact that speculators can coordinate better when they have common information about the central bank's signal, since this information is very revealing about the central bank's action.

Finally, we would like to stress that the negative effect of transparency on policy is due to the fact that the central bank releases information which becomes common knowledge to all speculators. One could imagine a different form of transparency, by which the central bank releases information that is interpreted differently by different speculators. In such a case, transparency increases the precision of speculators' private signals and the above conclusion is overturned. Thus, our model has strong implications regarding the optimal form of transparency.

The next proposition analyzes the second case and shows that if the additional signal provided by the central bank has a noise term that is specific to each speculator, then transparency improves the precision of private information for each speculator and weakens the incentive for them to coordinate.

Proposition 9 Suppose that the central bank releases a public signal, which is interpreted differently by different agents. Specifically, each agent observes $s_{a i}=s_{b}+\sigma_{a} \epsilon_{a i}$ where $\epsilon_{a i} \sim$ $N(0,1)$ and drawn independently across speculators. The weight that the speculators put on $s_{p}$ in the unique linear threshold equilibrium is smaller than the one characterized in Proposition 1.

\section{PROOF OF PROPOSITION 9:}

Central bank observes $s_{b}$, and speculators observe $s_{a i}, s_{p}$ and $s_{i}$. Precision of $s_{a i}$ is $\tau_{a h}=$ 
$1 /\left(\sigma_{b}^{2}+\sigma_{a}^{2}\right)$. The variance of the speculator's private information about $\theta$ (that is, two combined private signals $)$ is $1 /\left(\tau_{s}+\tau_{a h}\right)$, smaller than $1 / \tau_{s}$. Following steps that are similar to those in the proof of Proposition 1 we find that the equilibrium weight $k_{t r}^{\prime}$ put by speculators on $s_{p}$ in the unique linear threshold equilibrium satisfies

$$
-\frac{\tau_{b}\left(\tau_{s}+\tau_{a h}\right)}{\tau_{p}}\left(k_{t r}^{\prime}\right)^{3}+\left(\tau_{b}+\tau_{p}\right)\left(k_{t r}^{\prime}\right)^{2}+2 \tau_{p} k_{t r}^{\prime}+\tau_{p}=0 .
$$

(There are two differences between the derivations of equation (3) in Proposition 1 and the equation above. First, $\tau_{s}$ is replaced with $\tau_{s}+\tau_{a h}$. Second, for the speculators $\theta$ and $\epsilon_{b}$ are no longer idependently distributed. However, this second difference does not impact the derivation in a substantial way.) The result then follows from equation (3).

\section{Conclusion}

We analyze a model where the information revealed in the course of a speculative attack is used by the central bank in its policy decisions. On the one hand, this information enhances the effectiveness of the central bank's policy decisions. On the other hand, the fact that the central bank uses the information gives rise to endogenous strategic complementarities - which we call informational complementarities - due to which speculators wish to coordinate on similar trading actions even if they conflict with their private information. These coordination motives reduce the informational content of the speculators' collective action and the effectiveness of the central bank policy decisions. We analyze the tradeoff between information and coordination in speculative attacks, and derive comparative statics regarding the behavior of speculators and the effectiveness of the central bank's policy decisions. We also analyze the effect of different policy measures that the central bank may adopt to improve the effectiveness of its decisions.

Overall, the contribution of our paper is twofold. First, we introduce an important channel to the literature on currency attacks - namely, the learning by the central bank from market activities. We show the positive and negative aspects of such learning and study the nature of speculative attacks that it generates. Second, we provide a new angle to the literature on the feedback effect from financial markets to the real economy. We show that the fact that a decision maker learns from the trading process and takes an action that affects the value of traded securities gives rise to coordination problems among market participants, which result in destabilizing trading and reduced policy effectiveness. Thus, 
the analysis here can be used to study other settings where decision makers learn from the aggregate trade in financial markets. Examples include learning by firm managers and providers of capital when deciding whether to go ahead with an investment project. 


\section{Appendix A}

\section{Proofs}

\section{PROOF OF PROPOSITION 6:}

Inspecting the expressions for the probability of a policy mistake in Proposition 4, we see that in order to prove the current proposition, we need to show that $\tau_{T}$ increases in $\tau_{b}$ and $\tau_{s}$, decreases in $\tau_{p}$ if $0<\tau_{p}<\tau_{b} \frac{\sqrt{1+16 \frac{\tau_{s}}{\tau_{b}}}-1}{8}$ and increases in $\tau_{p}$ if $\tau_{p}>\tau_{b} \frac{\sqrt{1+16 \frac{\tau_{s}}{\tau_{b}}}-1}{8}$.

From Equation (5), note that

$$
\frac{\partial \tau_{T}}{\partial \tau_{s}}=-2 \tau_{p} \frac{(1+k)}{k^{2}} \frac{1}{k} \frac{\partial k}{\partial \tau_{s}}>0 \text { and } \frac{\partial \tau_{T}}{\partial \tau_{b}}=-2 \tau_{p} \frac{(1+k)}{k^{2}} \frac{1}{k} \frac{\partial k}{\partial \tau_{b}}>0 .
$$

Next note,

$$
\frac{\partial \tau_{T}}{\partial \tau_{p}}=\frac{(1+k)}{k^{2}}\left[(1+k)-2 \tau_{p} \frac{1}{k} \frac{\partial k}{\partial \tau_{p}}\right]
$$

and from Equation (3),

$$
\frac{\partial k}{\partial \tau_{p}}=\frac{\frac{\tau_{b} \tau_{s}}{\tau_{p}^{2}} k^{3}+k^{2}+2 k+1}{3 \frac{\tau_{b} \tau_{s}}{\tau_{p}} k^{2}-2\left(\tau_{b}+\tau_{p}\right) k-2 \tau_{p}} .
$$

Substituting and rearranging we obtain:

$$
\frac{\partial \tau_{T}}{\partial \tau_{p}}=\frac{(1+k)}{k^{2}}\left[\frac{3 \frac{\tau_{b} \tau_{s}}{\tau_{p}} k^{4}+\frac{\tau_{b} \tau_{s}}{\tau_{p}} k^{3}-2\left(\tau_{b}+\tau_{p}\right) k^{3}-2\left(\tau_{b}+\tau_{p}\right) k^{2}-4 \tau_{p} k^{2}-6 \tau_{p} k-2 \tau_{p}}{3 \frac{\tau_{b} \tau_{s}}{\tau_{p}} k^{3}-2\left(\tau_{b}+\tau_{p}\right) k^{2}-2 \tau_{p} k}\right] .
$$

We know that $k>0$. Moreover, we know from the proof of Proposition 3 that $3 \frac{\tau_{b} \tau_{s}}{\tau_{p}} k^{3}-$ $2\left(\tau_{b}+\tau_{p}\right) k^{2}-2 \tau_{p} k>0$. So the sign of $\frac{\partial \tau_{T}}{\partial \tau_{p}}$ is the same as the sign of

$$
3 \frac{\tau_{b} \tau_{s}}{\tau_{p}} k^{4}+\frac{\tau_{b} \tau_{s}}{\tau_{p}} k^{3}-2\left(\tau_{b}+\tau_{p}\right) k^{3}-2\left(\tau_{b}+\tau_{p}\right) k^{2}-4 \tau_{p} k^{2}-6 \tau_{p} k-2 \tau_{p} .
$$

We can rewrite this expression as:

$$
\begin{aligned}
& 3 \frac{\tau_{b} \tau_{s}}{\tau_{p}} k^{4}-3\left(\tau_{b}+\tau_{p}\right) k^{3}-6 \tau_{p} k^{2}-3 \tau_{p} k \\
& +\frac{\tau_{b} \tau_{s}}{\tau_{p}} k^{3}+\left(\tau_{b}+\tau_{p}\right) k^{3}+2 \tau_{p} k^{2}-2\left(\tau_{b}+\tau_{p}\right) k^{2}-3 \tau_{p} k-2 \tau_{p} .
\end{aligned}
$$

The first line of this expression is zero from Equation (3). We can rewrite the second line as:

$$
\begin{aligned}
& \frac{\tau_{b} \tau_{s}}{\tau_{p}} k^{3}-\left(\tau_{b}+\tau_{p}\right) k^{2}-2 \tau_{p} k-\tau_{p} \\
& +\left(\tau_{b}+\tau_{p}\right) k^{3}+2 \tau_{p} k^{2}-\left(\tau_{b}+\tau_{p}\right) k^{2}-\tau_{p} k-\tau_{p} .
\end{aligned}
$$

Once again the first line is zero by Equation (3). The second line can be rewritten as:

$$
(k-1)\left(\tau_{b} k^{2}+\tau_{p}(k+1)^{2}\right) .
$$


Thus the sign of $\frac{\partial \tau_{T}}{\partial \tau_{p}}$ is the same as the sign of $(k-1)$. To determine the sign of $(k-1)$, we evaluate the left side of Equation (3) at $k=1$ which is

$$
\frac{4 \tau_{p}^{2}+\tau_{b} \tau_{p}-\tau_{b} \tau_{s}}{\tau_{p}} .
$$

If the value of the above expression is positive then $k>1$, and if it is negative then $k<1$. (This is because the cubic function on left side of Equation (3) crosses zero only once and from above.) It is easy to see that the above expression is negative if

$$
0<\tau_{p}<\tau_{b} \frac{\sqrt{1+16 \frac{\tau_{s}}{\tau_{b}}}-1}{8}
$$

and positive if

$$
\tau_{p}>\tau_{b} \frac{\sqrt{1+16 \frac{\tau_{s}}{\tau_{b}}}-1}{8} .
$$

Thus the sign of $\frac{\partial \tau_{T}}{\partial \tau_{p}}$ is negative if $0<\tau_{p}<\tau_{b} \frac{\sqrt{1+16 \frac{\tau_{s}}{\tau_{b}}}-1}{8}$ and positive if $\tau_{p}>\tau_{b} \frac{\sqrt{1+16 \frac{\tau_{s}}{\tau_{b}}}-1}{8}$.

\section{PROOF OF PROPOSITION 7:}

Suppose that speculators follow linear strategies, that is, a speculator attacks if and only if his signal $s_{i}$ is below $s(0)-k s_{p}$. As before, the central bank observes:

$$
\frac{s(0)-\sigma_{s} T}{1+k}=\theta+\frac{k \sigma_{p}}{1+k} \epsilon_{p} .
$$

Suppose that the central bank abandons the status quo if and only if

$$
\left(\frac{\tau_{T}-\beta}{\tau_{T}+\tau_{b}}\right)\left(\frac{\hat{s}(0)-\sigma_{s} T}{1+k}\right)+\left(\frac{\tau_{b}+\beta}{\tau_{T}+\tau_{b}}\right) s_{b} \leq 0
$$

or

$$
T \geq \frac{1}{\sigma_{s}} s(0)+\frac{(1+k)}{\sigma_{s}} \frac{\left(\tau_{b}+\beta\right)}{\left(\tau_{T}-\beta\right)} s_{b}
$$

for $\tau_{T}>\beta>0$ where $\tau_{T}=\tau_{p} \frac{(1+k)^{2}}{k^{2}}$. Here, $\beta$ is the deviation from ex-post optimal weights. It measures the increase (decrease) in the weight given to the central bank's private signal (to the information in the attack) relative to the ex-post optimal level. The posterior belief 
of the regime change for a speculator with signal $s$ and $s_{p}$ is now expressed as follows:

$$
\begin{aligned}
& \operatorname{Pr}\left(T \geq \frac{1}{\sigma_{s}} s(0)+\frac{(1+k)}{\sigma_{s}} \frac{\left(\tau_{b}+\beta\right)}{\left(\tau_{T}-\beta\right)} s_{b} \mid s_{i}, s_{p}\right) \\
= & \Phi\left(\frac{-\left(1+(1+k) \frac{\left(\tau_{b}+\beta\right)}{\left(\tau_{T}-\beta\right)}\right) \frac{\tau_{s}}{\tau_{s}+\tau_{p}} s_{i}-\left(\left((1+k) \frac{\left(\tau_{b}+\beta\right)}{\left(\tau_{T}-\beta\right)}+1\right) \frac{\tau_{p}}{\tau_{s}+\tau_{p}}+k\right) s_{p}}{\sqrt{\left((1+k) \frac{\left(\tau_{b}+\beta\right)}{\left(\tau_{T}-\beta\right)}+1\right)^{2} \frac{1}{\tau_{s}+\tau_{p}}+\left((1+k) \frac{\left(\tau_{b}+\beta\right)}{\left(\tau_{T}-\beta\right)} \sigma_{b}\right)^{2}}}\right)
\end{aligned}
$$

For the proposed linear strategies to be best response, the following equation must hold for a speculator at the cutoff for all $s_{p}$ :

$$
c=\Phi\left(\frac{-\left((1+k) \frac{\left(\tau_{b}+\beta\right)}{\left(\tau_{T}-\beta\right)}+1\right) \frac{\tau_{s}}{\tau_{s}+\tau_{p}} s(0)-\left(\left((1+k) \frac{\left(\tau_{b}+\beta\right)}{\left(\tau_{T}-\beta\right)}+1\right)\left(\frac{\tau_{p}}{\tau_{s}+\tau_{p}}-\frac{\tau_{s}}{\tau_{s}+\tau_{p}} k\right)+k\right) s_{p}}{\sqrt{\left((1+k) \frac{\left(\tau_{b}+\beta\right)}{\left(\tau_{T}-\beta\right)}+1\right)^{2} \frac{1}{\tau_{s}+\tau_{p}}+\left((1+k) \frac{\left(\tau_{b}+\beta\right)}{\left(\tau_{T}-\beta\right)} \sigma_{b}\right)^{2}}}\right) .
$$

Thus for the linear strategy to be a best response, the coefficient of $s_{p}$ must be zero. In other words, $k$ must satisfy:

$$
-\frac{\tau_{s}}{\tau_{s}+\tau_{p}} k+\frac{\tau_{p}}{\tau_{s}+\tau_{p}}+\frac{k}{(1+k) \frac{\left(\tau_{b}+\beta\right)}{\left(\tau_{T}-\beta\right)}+1}=0
$$

Substituting for $\tau_{T}$ and rearranging the above equation we obtain:

$$
\frac{-\beta \tau_{s}-\tau_{b} \tau_{s}}{\tau_{p}} k^{3}+k^{2}\left(\tau_{p}+\tau_{b}\right)+2 k \tau_{p}+\tau_{p}=0 .
$$

For $\beta>0$ close to zero the above equation has a unique positive root and

$$
\frac{\partial k}{\partial \beta}=-3 \frac{\tau_{s} k}{\beta \tau_{s}+\tau_{b} \tau_{s}}<0 .
$$

Furthermore,

$$
\begin{aligned}
\frac{\partial \tau_{T}}{\partial \beta} & =2 \tau_{p}\left(\frac{(1+k)}{k^{2}}-\frac{(1+k)^{2}}{k^{3}}\right) \frac{\partial k}{\partial \beta}=-6 \tau_{p}\left(\frac{(1+k)}{k^{2}}-\frac{(1+k)^{2}}{k^{3}}\right) \frac{\tau_{s} k}{\beta \tau_{s}+\tau_{b} \tau_{s}} \\
& =6 \tau_{p}\left(\frac{1+k}{k^{2}}\right) \frac{\tau_{s}}{\beta \tau_{s}+\tau_{b} \tau_{s}}>0 .
\end{aligned}
$$

Thus increasing $\beta$ always leads to a more informative attack.

Next, we compute the ex-ante probability of abandoning the status quo for a given $\theta$ :

$$
\begin{aligned}
& \operatorname{Pr}\left(T \geq \frac{1}{\sigma_{s}} s(0)+\frac{(1+k)}{\sigma_{s}} \frac{\left(\tau_{b}+\beta\right)}{\left(\tau_{T}-\beta\right)} s_{b} \mid \theta\right) \\
= & \Phi\left(-\frac{1}{\sigma_{b}} \frac{\left(1+\frac{\left(\tau_{T}-\beta\right)}{\left(\tau_{b}+\beta\right)}\right)}{\sqrt{1+\frac{\tau_{b}\left(\tau_{T}-\beta\right)^{2}}{\tau_{T}\left(\tau_{b}+\beta\right)^{2}}}} \theta\right)=\Phi\left(-\frac{1}{\sigma_{b}}\left(\frac{\tau_{b} \tau_{T}+\tau_{T}^{2}}{\tau_{b} \tau_{T}+\beta^{2}}\right)^{\frac{1}{2}} \theta\right) .
\end{aligned}
$$


Moreover,

$$
\begin{aligned}
\frac{\partial}{\partial \beta}\left(\frac{\tau_{b} \tau_{T}+\tau_{T}^{2}}{\tau_{b} \tau_{T}+\beta^{2}}\right) & =\frac{\tau_{b} \frac{\partial \tau_{T}}{\partial \beta}+2 \tau_{T} \frac{\partial \tau_{T}}{\partial \beta}}{\tau_{b} \tau_{T}+\beta^{2}}-\frac{\tau_{b} \tau_{T}+\tau_{T}^{2}}{\left(\tau_{b} \tau_{T}+\beta^{2}\right)^{2}}\left(\tau_{b} \frac{\partial \tau_{T}}{\partial \beta}+2 \beta\right) \\
& =\frac{1}{\tau_{b} \tau_{T}+\beta^{2}}\left(\left(\frac{\tau_{b} \tau_{T}^{2}+2 \tau_{T} \beta^{2}+\tau_{b} \beta^{2}}{\tau_{b} \tau_{T}+\beta^{2}}\right) \frac{\partial \tau_{T}}{\partial \beta}-\frac{\tau_{b} \tau_{T}+\tau_{T}^{2}}{\left(\tau_{b} \tau_{T}+\beta^{2}\right)} 2 \beta\right) .
\end{aligned}
$$

Computing at $\beta=0$,

$$
\left.\frac{\partial}{\partial \beta}\left(\frac{\tau_{b} \tau_{T}+\tau_{T}^{2}}{\tau_{b} \tau_{T}+\beta^{2}}\right)\right|_{\beta=0}=\left.\frac{1}{\tau_{b}} \frac{\partial \tau_{T}}{\partial \beta}\right|_{\beta=0}>0 .
$$

Thus, for $\theta>0(\theta<0)$ the ex-ante probability of abandoning the status quo decreases (increases) if the central bank sets $\beta$ slightly larger than zero.

\section{PROOF OF PROPOSITION 8:}

Central bank observes both $s_{b}$ and $s_{a}$, and speculators only observe $s_{a}, s_{p}$ and $s_{i}$. We construct a linear equilibrium in which a speculator attacks if and only if $s_{i} \leq \hat{s}(0)-$ $m s_{a}-k_{t r} s_{p}$ where $m>0, k_{t r}>0$. The size of the attack from speculators given $\theta, s_{p}$ and $s_{a}$ is $A\left(\theta, s_{p}\right)=\Phi\left(\frac{\hat{s}(0)-m s_{a}-k_{t r} s_{p}-\theta}{\sigma_{s}}\right)$. The central bank observes $T\left(\theta, s_{p}\right)=\Phi^{-1}(A)$, or equivalently, it observes

$$
T=\frac{\hat{s}(0)-m s_{a}-k_{t r} s_{p}-\theta}{\sigma_{s}}
$$

which can be rewritten as:

$$
\frac{\hat{s}(0)-m s_{a}-\sigma_{s} T}{1+k_{t r}}=\theta+\frac{k_{t r} \sigma_{p} \epsilon_{p}}{1+k_{t r}} .
$$

Thus, the precision of the attack as a signal of the fundamental is

$$
\tau_{T}=\frac{\tau_{p}\left(1+k_{t r}\right)^{2}}{k_{t r}^{2}}
$$

and

$$
E\left[\theta \mid T, s_{b}, \epsilon_{a}\right]=\frac{\tau_{T}}{\tau_{T}+\tau_{b}}\left(\frac{\hat{s}(0)-m\left(s_{b}+\sigma_{a} \epsilon_{a}\right)-\sigma_{s} T}{1+k_{t r}}\right)+\frac{\tau_{b}}{\tau_{T}+\tau_{b}} s_{b} .
$$

This implies the status quo is abandoned if and only if

$$
T \geq \frac{\hat{s}(0)}{\sigma_{s}}+\frac{1}{\sigma_{s}}\left(\frac{\tau_{b}}{\tau_{T}}\left(1+k_{t r}\right)-m\right) s_{b}-\frac{1}{\sigma_{s}} m \sigma_{a} \epsilon_{a} .
$$

The precision of $s_{a}$ as a signal for $\theta$ is

$$
\tau_{a}=\frac{1}{\sigma_{b}^{2}+\sigma_{a}^{2}} .
$$


Hence, for a speculator, $\theta$ is distributed with mean $\frac{\tau_{s}}{\tau_{s}+\tau_{p}+\tau_{a}} s_{i}+\frac{\tau_{p}}{\tau_{s}+\tau_{p}+\tau_{a}} s_{p}+\frac{\tau_{a}}{\tau_{s}+\tau_{p}+\tau_{a}} s_{a}$ and precision $\tau_{s}+\tau_{p}+\tau_{a}$. The posterior belief of the regime change for a speculator with signals $s_{i}, s_{p}$ and $s_{a}$ is expressed as follows:

$$
\begin{aligned}
& \operatorname{Pr}\left(T \geq \frac{\hat{s}(0)}{\sigma_{s}}+\frac{1}{\sigma_{s}}\left(\frac{\tau_{b}}{\tau_{T}}\left(1+k_{t r}\right)-m\right) s_{b}-\frac{1}{\sigma_{s}} m \sigma_{a} \epsilon_{a} \mid s_{i}, s_{p}, s_{a}\right) \\
= & \Phi\left(\frac{-m s_{a}-k_{t r} s_{p}-\left(1+\left(\frac{\tau_{b}}{\tau_{T}}\left(1+k_{t r}\right)-m\right)\right)\left(\frac{\tau_{s}}{\tau_{s}+\tau_{p}+\tau_{a}} s_{i}+\frac{\tau_{p}}{\tau_{s}+\tau_{p}+\tau_{a}} s_{p}+\frac{\tau_{a}}{\tau_{s}+\tau_{p}+\tau_{a}} s_{a}\right)}{\sqrt{\left(1+\left(\frac{\tau_{b}}{\tau_{T}}\left(1+k_{t r}\right)-m\right)\right)^{2} \frac{1}{\tau_{s}+\tau_{p}+\tau_{a}}+\left(\frac{\tau_{b}}{\tau_{T}}\left(1+k_{t r}\right)-m\right)^{2} \sigma_{b}^{2}+m^{2} \sigma_{a}^{2}}}\right)
\end{aligned}
$$

The agent with signal $\hat{s}(0)-m s_{a}-k_{t r} s_{p}$ must be indifferent between attacking or not:

$$
c=\Phi\left(\frac{-m s_{a}-k_{t r} s_{p}-\left(1+\left(\frac{\tau_{b}}{\tau_{T}}\left(1+k_{t r}\right)-m\right)\right)\left(\frac{\tau_{s}}{\tau_{s}+\tau_{p}+\tau_{a}}\left(\hat{s}(0)-m s_{a}-k_{t r} s_{p}\right)+\frac{\tau_{p} s_{p}+\tau_{a} s_{a}}{\tau_{s}+\tau_{p}+\tau_{a}}\right)}{\sqrt{\left(1+\left(\frac{\tau_{b}}{\tau_{T}}\left(1+k_{t r}\right)-m\right)\right)^{2} \frac{1}{\tau_{s}+\tau_{p}+\tau_{a}}+\left(\frac{\tau_{b}}{\tau_{T}}\left(1+k_{t r}\right)-m\right)^{2} \sigma_{b}^{2}+m^{2} \sigma_{a}^{2}}}\right) .
$$

Since in a linear equilibrium the coefficients on $s_{p}$ and $s_{b}$ must be zero, we obtain the following system of equations:

$$
\begin{array}{r}
-k_{t r}+\left(1+\left(\frac{\tau_{b}}{\tau_{T}}\left(1+k_{t r}\right)-m\right)\right) \frac{\tau_{s}}{\tau_{s}+\tau_{p}+\tau_{a}} k_{t r}-\left(1+\left(\frac{\tau_{b}}{\tau_{T}}\left(1+k_{t r}\right)-m\right)\right) \frac{\tau_{p}}{\tau_{s}+\tau_{p}+\tau_{a}}=0 \\
-m+\left(1+\left(\frac{\tau_{b}}{\tau_{T}}\left(1+k_{t r}\right)-m\right)\right) \frac{\tau_{s}}{\tau_{s}+\tau_{p}+\tau_{a}} m-\left(1+\left(\frac{\tau_{b}}{\tau_{T}}\left(1+k_{t r}\right)-m\right)\right) \frac{\tau_{a}}{\tau_{s}+\tau_{p}+\tau_{a}}=0
\end{array}
$$

To solve for $k_{t r}$ and $m$, by rearranging the two equations and take the ratio of both sides, we obtain

$$
m=\frac{\tau_{a}}{\tau_{p}} k_{t r}
$$

Substituting this into the first equation gives:

$$
\tau_{s}\left(\frac{\tau_{b}-\tau_{a}}{\tau_{p}}\right) k_{t r}^{3}+\left(\tau_{s}\left(\frac{\tau_{p}-\tau_{a}}{\tau_{p}}\right)-\tau_{s}-\tau_{p}-\tau_{b}\right) k_{t r}^{2}-2 \tau_{p} k_{t r}-\tau_{p}=0 .
$$

Notice that this equation boils down to the cubic equation we had before if we set $\tau_{a}=0$. Next we compute the discriminant:

$$
\Delta=\frac{4}{\tau_{p}^{2}}\left(\tau_{p} \tau_{b}+\tau_{a} \tau_{s}\right)\left(\tau_{p}^{2}+\tau_{b} \tau_{p}+\tau_{a} \tau_{s}\right)^{2}+\tau_{s}\left(\tau_{b}-\tau_{a}\right)\left(4 \tau_{p}^{2}+36 \tau_{b} \tau_{p}+9 \tau_{a} \tau_{s}+27 \tau_{b} \tau_{s}\right)>0 .
$$

Thus, there is a unique linear equilibrium. Moreover, in this equilibrium $k_{t r}$ and $m$ are both positive. To compare the weight $k_{t r}$ with the equilibrium weight $k$, that we obtained in Proposition 1, we evaluate equation (A1) at $k$ :

$\tau_{s}\left(\frac{\tau_{b}-\tau_{a}}{\tau_{p}}\right) k^{3}+\left(\tau_{s}\left(\frac{\tau_{p}-\tau_{a}}{\tau_{p}}\right)-\tau_{s}-\tau_{p}-\tau_{b}\right) k^{2}-2 \tau_{p} k-\tau_{p}=-\left(\frac{\tau_{s} \tau_{a}}{\tau_{p}}\right)\left(k^{3}+k^{2}\right)<0$.

This means that $k_{t r}>k$ 


\section{Appendix B}

\section{Coordination when Speculators Observe Correlated Private Signals}

Instead of assuming that speculators receive a common signal that is not observable to the central bank, we show in this appendix that the main result of the paper will go through with an information structure that does not assume such an extreme commonality.

Let us assume that speculator $i \in[0,1]$ receives two private signals, $s_{i}$ and $s_{p i}$. As before, the signal $s_{i}$ is of the form $s_{i}=\theta+\sigma_{s} \epsilon_{i}$ where $\epsilon_{i}$ is normally distributed with mean of zero and standard deviation of one, and the precision of the signal is denoted by $\tau_{s}=1 / \sigma_{s}^{2}$. The signal $s_{p i}$ is of the form $s_{p i}=\theta+\sigma_{p} \epsilon_{p}+\sigma_{h} \eta_{i}$ where $\epsilon_{p}$ and $\eta_{i}$ are both normally distributed with mean of zero and standard deviation of one. We let $\tau_{p}=1 / \sigma_{p}^{2}$, and $\tau_{h}=1 / \sigma_{h}^{2}$, so that the precision of $s_{p i}$ is $\tau_{h} \tau_{p} /\left(\tau_{h}+\tau_{p}\right)$. The key aspect of the information structure is that the signals $s_{p i}$ share a common noise term $\epsilon_{p}$, and hence are correlated across all speculators. This information structure is motivated by the notion that a part of the information generating process may be subject to common random shocks such as economy-wide common liquidity shocks. The information observed by the central bank remains the same as in the model in the main text. ${ }^{15}$ Again, we are not assuming that speculators individually are better informed than the central bank. In fact, each speculator's information might be much noisier than the central bank's information. The payoff and information structure are common knowledge. All error terms $-\epsilon_{b}, \epsilon_{p}, \epsilon_{i}$, and $\eta_{i}$ - are independent of each other and $\epsilon_{i}$ and $\eta_{i}$ are independent across investors.

We now formally define an equilibrium in this setting. Let $g\left(s_{i}, s_{p i}\right)$ denote the action of a speculator given the private signals $s_{i}$ and $s_{p i}, T\left(\theta, \epsilon_{p}\right)$ the size of the aggregate attack from speculators for given fundamental $\theta$ and the common noise term $\epsilon_{p}$, and $\delta\left(T, s_{b}\right)$ the action of the central bank as a function of the size of the attack and its signal. Furthermore, let $\nu\left(\theta \mid T, s_{b}\right)$ denote the posterior distribution of $\theta$ conditional on the central bank's information, and let $\mu\left(\theta \mid s_{i}, s_{p i}\right)$ denote the posterior distribution of $\theta$ conditional on a speculator's information.

Definition B1 An equilibrium consists of a strategy for the central bank, $\delta\left(T, s_{b}\right)$, a symmetric strategy for the agents, $g\left(s_{i}, s_{p i}\right)$, probability measures, $\nu\left(\cdot \mid T, s_{b}\right)$ and $\mu\left(\cdot \mid s_{i}, s_{p i}\right)$, such

\footnotetext{
${ }^{15}$ One may wonder why the central bank does not observe a similar signal with common noise $\epsilon_{p}$. As we will see below, in equilibrium, the central bank does observe a signal involving the common shock $\epsilon_{p}$ endogenously. Assuming that the central bank observes an exogenous signal with the common noise component would only complicate the analysis without changing the spirit of our results.
} 
that

$$
\begin{aligned}
& \delta\left(T, s_{b}\right) \in \operatorname{argmax}_{\delta \in\{0,1\}} \int_{-\infty}^{\infty} \delta \theta d \nu\left(\theta \mid T, s_{b}\right), \\
& g\left(s_{i}, s_{p i}\right) \in \operatorname{argmax}_{a \in\{0,1\}} a \cdot\left[\int_{-\infty}^{\infty} \int_{-\infty}^{\infty} \mathbf{1}_{\left[\delta\left(T\left(\theta, \epsilon_{p}\right), \theta+\sigma_{b} \epsilon_{b}\right)=0\right]} d \mu\left(\theta \mid s_{i}, s_{p i}\right) d \Phi\left(\epsilon_{b}\right)-c\right], \\
& T\left(\theta, \epsilon_{p}\right)=\Phi^{-1}\left(\int_{-\infty}^{\infty}\left(\int_{-\infty}^{\infty} g\left(\theta+\sigma_{s} \epsilon, \theta+\sigma_{p} \epsilon_{p}+\sigma_{h} \eta\right) \phi(\eta) d \eta\right) \phi(\epsilon) d \epsilon\right), \\
& \nu\left(\theta \mid T, s_{b}\right) \text { is obtained using Bayes' rule for any } T \text { and } s_{b}, \\
& \mu\left(\theta \mid s_{i}, s_{p i}\right) \text { is obtained using Bayes' rule for any } s_{i} \text { and } s_{p i} .
\end{aligned}
$$

Our focus will be on linear threshold equilibria. These are equilibria where speculators attack the currency if and only if their private signal $s_{i}$ is below a threshold $\hat{s}\left(s_{p i}\right)$, which is a linear function of the correlated private signal $s_{p i}$ and, the central bank abandons the regime if and only if the aggregate size of the attack $T$ is above a threshold $\hat{T}\left(s_{b}\right)$, which is also a linear function of its private signal $s_{b}$. The next proposition shows that there is a unique such equilibrium and characterizes it.

Proposition B1 There is a unique linear threshold equilibrium where the speculators' threshold strategy is:

$$
g\left(s_{i}, s_{p i}\right)= \begin{cases}1 & \text { if } \quad s_{i} \leq \hat{s}\left(s_{p i}\right) \\ 0 & \text { if } \quad s_{i}>\hat{s}\left(s_{p i}\right)\end{cases}
$$

and the central bank's strategy is

$$
\delta\left(T, s_{b}\right)=\left\{\begin{array}{lll}
1 & \text { if } \quad T \leq \hat{T}\left(s_{b}\right) \\
0 & \text { if } \quad T>\hat{T}\left(s_{b}\right)
\end{array} .\right.
$$

Here,

$$
\hat{s}\left(s_{p i}\right)=\hat{s}(0)-k s_{p i},
$$

where $k>0$ is the unique real root to the cubic equation:

$$
\begin{aligned}
& -\left(\frac{\tau_{s} \tau_{b}}{\tau_{p}}\right) k^{3}+\left(\left(\tau_{b}+\tau_{p}\right) \frac{\tau_{h}}{\left(\tau_{h}+\tau_{p}\right)}\right) k^{2}+\left(2 \tau_{p} \frac{\tau_{h}}{\left(\tau_{h}+\tau_{p}\right)}\right) k+\tau_{p} \frac{\tau_{h}}{\left(\tau_{h}+\tau_{p}\right)} \\
& -\left(\frac{\tau_{s} \tau_{p}}{\left(\tau_{h}+\tau_{p}\right)}\right)\left(k^{3}+2 k^{2}+k\right)=0,
\end{aligned}
$$

and

$$
\hat{T}\left(s_{b}\right)=\frac{1}{\sqrt{\sigma_{s}^{2}+k^{2} \sigma_{h}^{2}}}\left[\hat{s}(0)+(1+k) \frac{\tau_{b}}{\tau_{T}} s_{b}\right],
$$


where

$$
\tau_{T}=\tau_{p}\left(1+\frac{1}{k}\right)^{2}
$$

is the precision of the attack as a signal of the fundamental.

\section{PROOF OF PROPOSITION B1:}

Suppose an agent attacks if and only if $s_{i}+k s_{p i} \leq \hat{s}(0)$ where $k>0$. The size of the attack from speculators given $\theta$ and $\epsilon_{p}$ is $A\left(\theta, \epsilon_{p}\right)=\Phi\left(\frac{\hat{s}(0)-k \sigma_{p} \epsilon_{p}-(1+k) \theta}{\sqrt{\sigma_{s}^{2}+k^{2} \sigma_{h}^{2}}}\right)$. The central bank observes $T\left(\theta, \epsilon_{p}\right)=\Phi^{-1}(A)$, or equivalently, it observes

$$
T=\frac{\hat{s}(0)-k \sigma_{p} \epsilon_{p}-(1+k) \theta}{\sqrt{\sigma_{s}^{2}+k^{2} \sigma_{h}^{2}}},
$$

which can be rewritten as

$$
\frac{\hat{s}(0)-\sqrt{\sigma_{s}^{2}+k^{2} \sigma_{h}^{2}} T}{1+k}=\theta+\frac{k \sigma_{p}}{1+k} \epsilon_{p}
$$

Thus, the precision of the attack as a signal of the fundamental is

$$
\tau_{T}=\tau_{p}\left(1+\frac{1}{k}\right)^{2}
$$

and

$$
E\left[\theta \mid T, s_{b}\right]=\frac{\tau_{T}}{\tau_{T}+\tau_{b}}\left(\frac{\hat{s}(0)-\sqrt{\sigma_{s}^{2}+k^{2} \sigma_{h}^{2}} T}{1+k}\right)+\frac{\tau_{b}}{\tau_{T}+\tau_{b}} s_{b} .
$$

This implies the status quo is abandoned if and only if

$$
T \geq \frac{\hat{s}(0)}{\sqrt{\sigma_{s}^{2}+k^{2} \sigma_{h}^{2}}}+\frac{(1+k)}{\sqrt{\sigma_{s}^{2}+k^{2} \sigma_{h}^{2}}} \frac{\tau_{b}}{\tau_{T}} s_{b}=\hat{T}\left(s_{b}\right),
$$

which is Equation (B2).

For a speculator, $\theta$ is distributed with mean $\frac{\tau_{s}}{\tau_{s}+\tau_{p}^{\prime}} s_{i}+\frac{\tau_{p}^{\prime}}{\tau_{s}+\tau_{p}^{\prime}} s_{p i}$, where $\tau_{p}^{\prime}=\tau_{h} \tau_{p} /\left(\tau_{h}+\tau_{p}\right)$, and $\sigma_{h} \eta_{i}$ is distributed with mean $\frac{\tau_{p} \tau_{s}}{\tau_{s} \tau_{h}+\tau_{p} \tau_{h}+\tau_{p} \tau_{s}}\left(s_{p i}-s_{i}\right)$. Let $\Omega$ be the standard deviation of $\left[\left(1+(1+k) \frac{\tau_{b}}{\tau_{T}}\right) \theta+(1+k) \frac{\tau_{b}}{\tau_{T}} \sigma_{b} \epsilon_{b}-k \sigma_{h} \eta_{i}\right]$. Then, the posterior belief of the regime change for a speculator with signals $s_{i}$ and $s_{p i}$ is expressed as follows:

$$
\begin{aligned}
& \operatorname{Pr}\left(T \geq \frac{\hat{s}(0)}{\sqrt{\sigma_{s}^{2}+k^{2} \sigma_{h}^{2}}}+\frac{(1+k)}{\sqrt{\sigma_{s}^{2}+k^{2} \sigma_{h}^{2}}} \frac{\tau_{b}}{\tau_{T}} s_{b} \mid s_{i}, s_{p i}\right) \\
= & \operatorname{Pr}\left(\left(1+(1+k) \frac{\tau_{b}}{\tau_{T}}\right) \theta+(1+k) \frac{\tau_{b}}{\tau_{T}} \sigma_{b} \epsilon_{b}-k \sigma_{h} \eta_{i} \leq-k s_{p i} \mid s_{i}, s_{p i}\right) \\
= & \Phi\left(\frac{-k s_{p i}-\left(1+(1+k) \frac{\tau_{b}}{\tau_{T}}\right)\left(\frac{\tau_{s}}{\tau_{s}+\tau_{p}^{\prime}} s_{i}+\frac{\tau_{p}^{\prime}}{\tau_{s}+\tau_{p}^{\prime}} s_{p i}\right)+k \frac{\tau_{p} \tau_{s}}{\tau_{s} \tau_{h}+\tau_{p} \tau_{h}+\tau_{p} \tau_{s}}\left(s_{p i}-s_{i}\right)}{\Omega}\right) .
\end{aligned}
$$


Hence, in equilibrium, the threshold strategy must satisfy the following equation for all $s_{p i}$ :

$$
c=\Phi\left(\frac{\begin{array}{c}
\left(-\left(1+(1+k) \frac{\tau_{b}}{\tau_{T}}\right) \frac{\tau_{s}}{\tau_{s}+\tau_{p}^{\prime}}-k \frac{\tau_{p} \tau_{s}}{\tau_{s} \tau_{h}+\tau_{p} \tau_{h}+\tau_{p} \tau_{s}}\right)\left(\hat{s}(0)-k s_{p i}\right) \\
+\left(-k-\left(1+(1+k) \frac{\tau_{b}}{\tau_{T}}\right) \frac{\tau_{p}^{\prime}}{\tau_{s}+\tau_{p}^{\prime}}+k \frac{\tau_{p} \tau_{s}}{\tau_{s} \tau_{h}+\tau_{p} \tau_{h}+\tau_{p} \tau_{s}}\right) s_{p i}
\end{array}}{\Omega}\right) .
$$

Thus, for a linear equilibrium to exist the coefficient of $s_{p i}$ must be zero. In other words, $k$ must satisfy:

$$
\begin{aligned}
0= & -k\left(-\left(1+(1+k) \frac{\tau_{b}}{\tau_{T}}\right) \frac{\tau_{s}\left(\tau_{h}+\tau_{p}\right)}{\tau_{s} \tau_{h}+\tau_{s} \tau_{p}+\tau_{h} \tau_{p}}-k \frac{\tau_{p} \tau_{s}}{\tau_{s} \tau_{h}+\tau_{p} \tau_{h}+\tau_{p} \tau_{s}}\right) \\
& +\left(-k-\left(1+(1+k) \frac{\tau_{b}}{\tau_{T}}\right) \frac{\tau_{h} \tau_{p}}{\tau_{s} \tau_{h}+\tau_{s} \tau_{p}+\tau_{h} \tau_{p}}+k \frac{\tau_{p} \tau_{s}}{\tau_{s} \tau_{h}+\tau_{p} \tau_{h}+\tau_{p} \tau_{s}}\right) .
\end{aligned}
$$

Substituting $\tau_{T}$ into the above equation, we obtain

$$
\begin{aligned}
& \left(-\frac{\tau_{s}\left(\tau_{h}+\tau_{p}\right)}{\tau_{s} \tau_{h}+\tau_{s} \tau_{p}+\tau_{h} \tau_{p}} k+\frac{\tau_{h} \tau_{p}}{\tau_{p} \tau_{h}+\tau_{s} \tau_{p}+\tau_{h} \tau_{p}}\right)\left((1+k)+\frac{k^{2} \tau_{b}}{\tau_{p}}\right)+k(1+k) \\
& -k \frac{\tau_{s}}{\tau_{s} \tau_{h}+\tau_{p} \tau_{h}+\tau_{p} \tau_{s}}(1+k)^{2}=0 .
\end{aligned}
$$

By dividing the left side by $\left(\tau_{h}+\tau_{p}\right)$ and re-organized it, we find that equilibrium $k$ must satisfy the following equation:

$$
\begin{aligned}
& -\left(\frac{\tau_{s} \tau_{b}}{\tau_{p}}\right) k^{3}+\left(\left(\tau_{b}+\tau_{p}\right) \frac{\tau_{h}}{\left(\tau_{h}+\tau_{p}\right)}\right) k^{2}+\left(2 \tau_{p} \frac{\tau_{h}}{\left(\tau_{h}+\tau_{p}\right)}\right) k+\tau_{p} \frac{\tau_{h}}{\left(\tau_{h}+\tau_{p}\right)} \\
& -\left(\frac{\tau_{s} \tau_{p}}{\left(\tau_{h}+\tau_{p}\right)}\right)\left(k^{3}+2 k^{2}+k\right)=0,
\end{aligned}
$$

which is Equation (B1). Next, we show that there is a unique solution to the above equation with a positive $k$. Consider the following equation:

$$
-\left(\frac{\tau_{s} \tau_{b}}{\tau_{p}}\right) k^{3}+\left(\left(\tau_{b}+\tau_{p}\right) \frac{\tau_{h}}{\left(\tau_{h}+\tau_{p}\right)}\right) k^{2}+\left(2 \tau_{p} \frac{\tau_{h}}{\left(\tau_{h}+\tau_{p}\right)}\right) k+\tau_{p} \frac{\tau_{h}}{\left(\tau_{h}+\tau_{p}\right)}=0 .
$$

We compute the discriminant for Equation (B3):

$$
\begin{aligned}
\Delta= & \left(\frac{\tau_{h}}{\left(\tau_{h}+\tau_{p}\right)}\right)^{4}\left(\tau_{b}+\tau_{p}\right)^{2}\left(4 \tau_{b} \tau_{p}\right)+\left(\frac{\tau_{s} \tau_{b}}{\tau_{p}}\right)\left(\frac{\tau_{h}}{\left(\tau_{h}+\tau_{p}\right)}\right)^{3}\left(4 \tau_{p}^{3}+36 \tau_{b} \tau_{p}^{2}\right) \\
& +27\left(\frac{\tau_{s} \tau_{b}}{\tau_{p}}\right)^{2}\left(\tau_{p} \frac{\tau_{h}}{\left(\tau_{h}+\tau_{p}\right)}\right)^{2}>0 .
\end{aligned}
$$

Therefore, Equation (B3) has a unique real root. The left side of Equation (B3) goes to $\infty$ as $k$ goes to $-\infty$ and $-\infty$ as $k$ goes to $\infty$. Moreover, it is positive at $k=0$. Since the 
equation has a single real root, it must cross zero at a unique $k>0$. Next, consider the remaining term of Equation (B1):

$$
-\left(\frac{\tau_{s} \tau_{p}}{\tau_{h}+\tau_{p}}\right) k(k+1)^{2} .
$$

This term makes the left side of Equation (B1) shift up when $k<0$. Thus there can not be a root for the whole cubic equation when $k<0$. Moreover when $k>0$, this additional term makes the left side of equation (B1) shift down and decrease faster for every $k>0$. Thus, the left side of Equation (B1) can cross zero only once, and at $k>0$.

Proposition B2 The weight $k$ put by speculators on $s_{p i}$ in the unique linear threshold equilibrium characterized by Proposition B1 is greater than the weight $k_{B M}$ that would be put on $s_{p i}$ in a game where the central bank does not attempt to get information about $\theta$ from the size of the attack.

\section{PROOF OF PROPOSITION B2:}

We start by computing $k_{B M}$. When the central bank does not update its belief about $\theta$ based on the size of the attack, it will abandon the regime if and only if its private signal $s_{b}=\theta+\sigma_{b} \epsilon_{b}$ is negative. Then, speculators will attack the currency if and only if $\operatorname{Pr}\left(\theta+\sigma_{b} \epsilon_{b}<0 \mid s_{i}, s_{p i}\right)>c$.

In a linear threshold equilibrium, speculators attack if and only if their private signal $s_{i}$ is below the threshold $\hat{s}_{B M}\left(s_{p i}\right)=\hat{s}_{B M}(0)-k_{B M} s_{p i}$. Thus, the speculator who observes the threshold signal has to be indifferent between attacking and not attacking:

$$
\operatorname{Pr}\left(\theta+\sigma_{b} \epsilon_{b}<0 \mid \hat{s}_{B M}\left(s_{p i}\right), s_{p i}\right)=c .
$$

Since $\theta$ and $\epsilon_{b}$ are independently normally distributed, this implies that:

$$
\Phi\left(\frac{-\frac{\tau_{s}}{\tau_{s}+\tau_{p}^{\prime}} \hat{s}_{B M}\left(s_{p i}\right)-\frac{\tau_{p}^{\prime}}{\tau_{s}+\tau_{p}^{\prime}} s_{p i}}{\sqrt{\frac{1}{\tau_{s}+\tau_{p}^{\prime}}+\sigma_{b}^{2}}}\right)=c .
$$

Plugging in $\hat{s}_{B M}\left(s_{p i}\right)=\hat{s}_{B M}(0)-k_{B M} s_{p i}$, we get:

$$
\Phi\left(\frac{-\frac{\tau_{s}}{\tau_{s}+\tau_{p}^{\prime}} \hat{s}_{B M}(0)-\left(\frac{\tau_{p}^{\prime}}{\tau_{s}+\tau_{p}^{\prime}}-\frac{\tau_{s}}{\tau_{s}+\tau_{p}^{\prime}} k_{B M}\right) s_{p i}}{\sqrt{\frac{1}{\tau_{s}+\tau_{p}^{\prime}}+\sigma_{b}^{2}}}\right)=c .
$$

Since this equation has to hold for every $s_{p i}$, we get:

$$
k_{B M}=\frac{\tau_{p}^{\prime}}{\tau_{s}} .
$$


Now, we show that $k$ (defined in Equation (B1)) is greater than $k_{B M}$. To do this, we need to evaluate the left side of Equation (B1) at $k=k_{B M}=\frac{\tau_{p}^{\prime}}{\tau_{s}}=\frac{\tau_{h} \tau_{p}}{\left(\tau_{h}+\tau_{p}\right) \tau_{s}}$. This yields:

$$
\begin{aligned}
& -\left(\frac{\tau_{s} \tau_{b}}{\tau_{p}}\right)\left(\frac{\tau_{h} \tau_{p}}{\left(\tau_{h}+\tau_{p}\right) \tau_{s}}\right)^{3}+\left(\left(\tau_{b}+\tau_{p}\right) \frac{\tau_{h}}{\left(\tau_{h}+\tau_{p}\right)}\right)\left(\frac{\tau_{h} \tau_{p}}{\left(\tau_{h}+\tau_{p}\right) \tau_{s}}\right)^{2}+\left(2 \tau_{p} \frac{\tau_{h}}{\left(\tau_{h}+\tau_{p}\right)}\right) \frac{\tau_{h} \tau_{p}}{\left(\tau_{h}+\tau_{p}\right) \tau_{s}} \\
& +\tau_{p} \frac{\tau_{h}}{\left(\tau_{h}+\tau_{p}\right)}-\left(\frac{\tau_{s} \tau_{p}}{\left(\tau_{h}+\tau_{p}\right)}\right)\left(\left(\frac{\tau_{h} \tau_{p}}{\left(\tau_{h}+\tau_{p}\right) \tau_{s}}\right)^{3}+2\left(\frac{\tau_{h} \tau_{p}}{\left(\tau_{h}+\tau_{p}\right) \tau_{s}}\right)^{2}+\left(\frac{\tau_{h} \tau_{p}}{\left(\tau_{h}+\tau_{p}\right) \tau_{s}}\right)\right) .
\end{aligned}
$$

Re-organizing the terms, we get:

$$
=\frac{\tau_{h}^{2} \tau_{p}^{2}}{\left(\tau_{h}+\tau_{p}\right) \tau_{s}}\left(\frac{\tau_{p} \tau_{h}}{\left(\tau_{h}+\tau_{p}\right) \tau_{s}}\left(1-\frac{\tau_{p}}{\left(\tau_{h}+\tau_{p}\right)}\right)+2\left(1-\frac{\tau_{p}}{\tau_{h}+\tau_{p}}\right)\right)+\tau_{p} \tau_{h}\left(1-\frac{\tau_{p}}{\tau_{h}+\tau_{p}}\right)>0 .
$$

Hence, $k>k_{B M}$.

To sum up, we have demonstrated that the spirit of the equilibrium and the presence of informational complementarities remain when speculators observe correlated signals instead of a common signal.

\section{Appendix C}

\section{Common Signal about Noise Trading}

We now demonstrate that the central bank's policy decision is less efficient than the benchmark case even when speculators receive a common signal that is not about the fundamental. To illustrate this point suppose that there is some noise demand for the currency. Specifically, we assume that the central bank observes a noisy signal of the size of the attack from informed speculators, $T=\Phi^{-1}(A)+\sigma_{m} \epsilon_{m}$, where $A$ is the size of the aggregate attack from speculators, and the noise component of this signal, $\sigma_{m} \epsilon_{m}$, is normally distributed with a mean of zero and standard deviation $\sigma_{m} \cdot{ }^{16}$

Speculators' strategies are now functions of their private signals and the commonly observed noise level. Otherwise, equilibrium is defined analogously to the one in Definition 1. In this case, it is possible for speculators to coordinate on the level of noise demand and "fool" the central bank. The assumption on speculators' informational advantage about the noise trading level is motivated by the fact that although individual speculators may not know about the fundamental more than the central bank, they understand the institutional details or the workings of the currency market better. Under this assumption, we show that

\footnotetext{
${ }^{16}$ This specification is introduced by Dasgupta (2005).
} 
currency speculators trade more aggressively on their information when the noise component of currency attack is of a high level. As a result, the central bank cannot differentiate between a high noise attack or a low fundamental, which implies there might be occasions where the central bank on average abandons the status quo too often. We first state the result under these circumstances.

Proposition C1 There is a unique equilibrium where the speculators' threshold strategies are linear in $\epsilon_{m}$ such that

$$
g(s)=\left\{\begin{array}{ll}
1 & \text { if } \quad s_{i} \leq \bar{s}\left(\epsilon_{m}\right) \\
0 & \text { if } \quad s_{i}>\bar{s}\left(\epsilon_{m}\right)
\end{array},\right.
$$

and $\delta\left(T, s_{b}\right)=\left\{\begin{array}{ll}1 & \text { if } \quad T \leq \bar{T}\left(s_{b}\right) \\ 0 & \text { if } \quad T>\bar{T}\left(s_{b}\right)\end{array}\right.$, where $\bar{s}\left(\epsilon_{m}\right)=\bar{s}(0)+\bar{k} \epsilon_{m}$ where $\bar{k}>0$ is the unique real root of the cubic equation:

$$
\bar{k}^{3}+2 \sigma_{s} \sigma_{m} \bar{k}^{2}+\left(\sigma_{s} \sigma_{m}\right)^{2} \bar{k}-\sigma_{b}^{2} \sigma_{s} \sigma_{m}=0 .
$$

$\bar{s}(0)$ satisfies

$$
c=\Phi\left(\frac{-\left(1+\frac{\tau_{b}}{\bar{\tau}_{T}}\right) \bar{s}(0)}{\sqrt{\left(1+\frac{\tau_{b}}{\bar{\tau}_{T}}\right)^{2} \sigma_{s}^{2}+\left(\frac{\tau_{b}}{\bar{\tau}_{T}}\right)^{2} \sigma_{b}^{2}}}\right),
$$

and

$$
\bar{T}\left(s_{b}\right)=\frac{1}{\sigma_{s}}\left[\bar{s}(0)+\frac{\left(\bar{k}+\sigma_{s} \sigma_{m}\right)^{2}}{\sigma_{b}^{2}} s_{b}\right],
$$

where $\bar{\tau}_{T}=\frac{1}{\left(\bar{k}+\sigma_{s} \sigma_{m}\right)^{2}}$ is the precision of the attack as a signal of the fundamental.

\section{PROOF OF PROPOSITION C1:}

Suppose an agent attacks if and only if $s_{i} \leq \bar{s}\left(\epsilon_{m}\right)=\bar{s}(0)+\bar{k} \epsilon_{m}$. The size of the attack from speculators is $A\left(\theta, \epsilon_{m}\right)=\Phi\left(\frac{\bar{s}\left(\epsilon_{m}\right)-\theta}{\sigma_{s}}\right)$. The central bank observes $T\left(\theta, \epsilon_{m}\right)=\Phi^{-1}(A)+\sigma_{m} \epsilon_{m}$, or equivalently, it observes

$$
\begin{aligned}
T & =\frac{\bar{s}(0)+\bar{k} \epsilon_{m}-\theta}{\sigma_{s}}+\sigma_{m} \epsilon_{m}, \\
\bar{s}(0)-\sigma_{s} T & =\theta-\left(\bar{k}+\sigma_{s} \sigma_{m}\right) \epsilon_{m}
\end{aligned}
$$

and

$$
E\left[\theta \mid T, s_{b}\right]=\frac{\bar{\tau}_{T}}{\bar{\tau}_{T}+\tau_{b}}\left(\bar{s}(0)-\sigma_{s} T\right)+\frac{\tau_{b}}{\bar{\tau}_{T}+\tau_{b}} s_{b}
$$


where $\bar{\tau}_{T}=\frac{1}{\left(\bar{k}+\sigma_{s} \sigma_{m}\right)^{2}}$. This implies the status quo is abandoned if and only if

$$
T \geq \frac{1}{\sigma_{s}}\left[\bar{s}(0)+\frac{\tau_{b}}{\bar{\tau}_{T}} s_{b}\right]=\bar{T}\left(s_{b}\right),
$$

which is Equation (C3).

The posterior belief of the regime change for a speculator with signal $s_{i}$ and $\epsilon_{m}$ is expressed as follows:

$$
\operatorname{Pr}\left(T \geq \frac{1}{\sigma_{s}}\left[\bar{s}(0)+\frac{\tau_{b}}{\bar{\tau}_{T}} s_{b}\right] \mid s_{i}, \epsilon_{m}\right)=\Phi\left(\frac{\left(\bar{k}+\sigma_{s} \sigma_{m}\right) \epsilon_{m}-\left(1+\frac{\tau_{b}}{\bar{\tau}_{T}}\right) s_{i}}{\sqrt{\left(1+\frac{\tau_{b}}{\bar{\tau}_{T}}\right)^{2} \sigma_{s}^{2}+\left(\frac{\tau_{b}}{\bar{\tau}_{T}}\right)^{2} \sigma_{b}^{2}}}\right) .
$$

Hence, $\bar{s}\left(\epsilon_{m}\right)=\bar{s}(0)+k \epsilon_{m}$ must solve

$$
c=\Phi\left(\frac{-\left(1+\frac{\tau_{b}}{\bar{\tau}_{T}}\right) \bar{s}(0)+\left(\sigma_{s} \sigma_{m}-\bar{k} \frac{\tau_{b}}{\bar{\tau}_{T}}\right) \epsilon_{m}}{\sqrt{\left(1+\frac{\tau_{b}}{\bar{\tau}_{T}}\right)^{2} \sigma_{s}^{2}+\left(\frac{\tau_{b}}{\bar{\tau}_{T}}\right)^{2} \sigma_{b}^{2}}}\right) .
$$

To solve this equation for all $\epsilon_{m}$, the coefficient of $\epsilon_{m}$ must be zero. In other words,

$$
\sigma_{s} \sigma_{m}-\bar{k} \frac{\tau_{b}}{\bar{\tau}_{T}}=0
$$

Rearranging we find that $\bar{k}$ must solve:

$$
\bar{k}^{3}+2 \sigma_{s} \sigma_{m} \bar{k}^{2}+\left(\sigma_{s} \sigma_{m}\right)^{2} \bar{k}-\sigma_{b}^{2} \sigma_{s} \sigma_{m}=0 .
$$

To see that this equation has a unique real root we compute the discriminant $\Delta$ of the cubic equation:

$$
\Delta=4\left(\sigma_{s} \sigma_{m}\right)^{4} \frac{1}{\tau_{b}}+27\left(\frac{\sigma_{s} \sigma_{m}}{\tau_{b}}\right)^{2}>0 .
$$

Since $\Delta>0$ the equation has a unique real root. Moreover, the left-hand side of equation (C6) goes to $-\infty$ as $\bar{k}$ goes to $-\infty$ and it is negative at $\bar{k}=0$. Since the equation has a single real root, it must cross zero at some $\bar{k}>0$. Given $\bar{k}$, we obtain $\bar{s}(0)$ as the solution to Equation (C2).

In other words, the equilibrium strategy for the central bank is to abandon the exchange regime if and only if the observed signal of aggregate attack, $T$, is greater than or equal to the threshold, $\bar{T}\left(s_{b}\right)$, a function of the central bank's private signal, $s_{b}$. The equilibrium strategy for a speculator who receives a signal, $s$, is to attack if and only if $s$ falls below a threshold value, $\bar{s}\left(\epsilon_{m}\right)$, which is a linear increasing function of the noise trading level in the 
currency market.

Proposition C2 The weight $\bar{k}$ put by speculators on $\epsilon_{m}$ in the unique linear threshold equilibrium characterized by Proposition $C 1$ is greater than the weight $\bar{k}_{B M}$ that would be put on $\epsilon_{m}$ in a game where the central bank does not attempt to get information about $\theta$ from the size of the attack.

The proof is straightforward since $\bar{k}$ is strictly positive and if the central bank does not infer the fundamental from $T, \bar{k}_{B M}=0$.

Empirically, it has been difficult to explain the fact that speculative attacks tend to be contagious even though the fundamentals of the countries under attack are quite different. Our finding in this section offers an explanation. Since the noise trading level tends to be high across countries during the periods of market upheavals such as the ERM debacles (1992), the Asian crisis (1997), and the Russian default (1998), currency speculators, knowing so, coordinate their attacks across countries around these times. Given this finding, central banks should adjust their behavior during contagious speculative attacks - they should rely less on market information to make policy decisions.

\section{Appendix D}

\section{Speculators Observe Fundamental Perfectly}

We begin by defining equilibrium in this setting. Let $A(\theta)$ denote the size of the aggregate attack given $\theta, g(\theta)$ the action of an agent given $\theta$, and $\delta\left(A, s_{b}\right)$ the action of the central bank as a function of the size of the attack and its signal. Furthermore, let $\nu\left(\theta \mid A, s_{b}\right)$ denote the posterior belief by the central bank conditional on $A$ and $s_{b}$.

Definition D1 An equilibrium consists of a mapping A from the fundamental to the size of the attack, a strategy for the central bank, $\delta\left(A, s_{b}\right)$, a symmetric strategy for the agents, $g(\theta)$ and a probability measure, $\nu\left(\cdot \mid A, s_{b}\right)$, such that

$$
\begin{aligned}
& \delta\left(A, s_{b}\right) \in \operatorname{argmax}_{\delta \in\{0,1\}} \int_{-\infty}^{\infty} \delta \theta d \nu\left(\theta \mid A, s_{b}\right), \\
& g(\theta) \in \operatorname{argmax}_{a \in\{0,1\}} a \cdot\left[\int_{-\infty}^{\infty} \mathbf{1}_{\left[\delta\left(A(\theta), \theta+\sigma_{b} \epsilon_{b}\right)=0\right]} d \phi\left(\epsilon_{b}\right)-c\right] \\
& \nu\left(\theta \mid A, s_{b}\right) \text { is obtained using Bayes' rule for any } A, s_{b}, \\
& A(\theta)=1 \text { if } g(\theta)=1 \text { and } A(\theta)=0 \text { otherwise. }
\end{aligned}
$$


Since speculators have information that is valuable to the central bank, in equilibrium, the central bank would make its policy decision based on the information revealed through speculators' actions as well as its own noisy private signal. When the fundamental is common knowledge among them, speculators may coordinate in various ways leading to different inferences by the central bank, resulting in multiple equilibria. In particular, speculators may be able to convince the central bank to abandon the fixed exchange rate regime even when it is not optimal for the central bank to do so. Our next proposition shows that multiplicity may arise when the speculators follow symmetric cut-off strategies.

Proposition D1 There exists $\theta^{*}>0$ such that for all $\bar{\theta} \in\left[0, \theta^{*}\right]$ there is an equilibrium where $g(\theta)=1$ if $\theta \leq \bar{\theta}$ and $g(\theta)=0$ if $\theta>\bar{\theta}$.

\section{PROOF OF PROPOSITION D1:}

Suppose $g(\theta)=1$ if $\theta \leq \bar{\theta}$ and $g(\theta)=0$ if $\theta>\bar{\theta}$. Hence, the expectation of $\theta$ conditional on observing $A=1$ and $s_{b}$ is

$$
E\left[\theta \mid \theta \leq \bar{\theta}, s_{b}\right]=\frac{\int_{-\infty}^{\bar{\theta}} \theta \phi\left(\frac{\theta-s_{b}}{\sigma_{b}}\right) d \theta}{\Phi\left(\frac{\bar{\theta}-s_{b}}{\sigma_{b}}\right)} .
$$

Next, we show that $E\left[\theta \mid \theta \leq \bar{\theta}, s_{b}\right]$ is increasing in $s_{b}$. To see this, note that $E\left[\theta \mid \theta \leq \bar{\theta}, s_{b}+\Delta\right]=$ $\Delta-E\left[\theta \mid \theta \leq \bar{\theta}-\Delta, s_{b}\right]$. Subtracting $E\left[\theta \mid \theta \leq \bar{\theta}, s_{b}\right]$ from both sides, dividing by $\Delta$ and letting $\Delta$ go to zero, we obtain:

$$
\frac{\partial E\left[\theta \mid \theta \leq \bar{\theta}, s_{b}\right]}{\partial s_{b}}=1-\frac{\partial E\left[\theta \mid \theta \leq \bar{\theta}, s_{b}\right]}{\partial \bar{\theta}} .
$$

By Proposition 1 in Burdett (1996), we know that $\frac{\partial E\left[\theta \mid \theta \leq \bar{\theta}, s_{b}\right]}{\partial \bar{\theta}} \in[0,1]$. Thus, $\frac{\partial E\left[\theta \mid \theta \leq \bar{\theta}, s_{b}\right]}{\partial s_{b}} \geq 0$.

Suppose $\bar{\theta} \geq 0$. Since $\frac{\partial E\left[\theta \mid \theta \leq \bar{\theta}, s_{b}\right]}{\partial s_{b}} \geq 0$, there exists a $\hat{s}_{b}(\bar{\theta})>0$ (possibly infinite) such that

$$
E\left[\theta \mid \theta \leq \bar{\theta}, s_{b}\right]=\left\{\begin{array}{lll}
\geq 0 & \text { if } & s_{b} \geq \hat{s}_{b}(\bar{\theta}) \\
<0 & \text { if } & s_{b}<\hat{s}_{b}(\bar{\theta})
\end{array} .\right.
$$

Therefore, the central bank's strategy is

$$
\delta\left(A, s_{b}\right)=\left\{\begin{array}{cc}
1 & \text { if } \quad A=1 \text { and } s_{b}>\hat{s}_{b}(\bar{\theta}) \text { or } A=0 \\
0 & \text { o.w. }
\end{array} .\right.
$$

Moreover, $\hat{s}_{b}(\bar{\theta})$ is decreasing in $\bar{\theta}$.

Given the central bank's strategy and given the strategy of the other speculators, it is optimal for any speculator to set $g(\theta)=0$ if $\theta>\bar{\theta}$. Now, fix some $\theta \leq \bar{\theta}$. We want to show 
that if $\bar{\theta}<\theta^{*}$ for some $\theta^{*}>0$ then it is optimal for the speculators to set $g(\theta)=1$. Since in this case, $A=1$ and the probability that the central bank abandons the regime is $\Phi\left(\frac{\hat{s}_{b}(\bar{\theta})-\theta}{\sigma_{b}}\right)$. Note that this probability is decreasing in $\theta$, so if it is optimal for speculators to attack at $\bar{\theta}$, it is also optimal to attack at all smaller $\theta$. Now note that $\Phi\left(\frac{\hat{s}_{b}(\bar{\theta})-\bar{\theta}}{\sigma_{b}}\right)$ is one at $\bar{\theta}=0$, is continuous and decreases as $\bar{\theta}$ increases. Therefore, there is a threshold $\theta^{*}>0$ such that $\Phi\left(\frac{\hat{s}_{b}(\bar{\theta})-\bar{\theta}}{\sigma_{b}}\right)-c \gtreqless 0$ if $\bar{\theta} \lesseqgtr \theta^{*}$. Thus it is optimal to attack for the speculators if $\theta \leq \bar{\theta}$ where $0 \leq \bar{\theta} \leq \theta^{*}$. This proves the proposition.

In other words, this proposition shows that there are multiple equilibria in which the speculators follow a cut-off strategy: They attack the currency regime when their signal is below $\bar{\theta}$ and do not attack otherwise. The cut-off value could be any $\theta$ between $\left[0, \theta^{*}\right]$. This means that the central bank may devalue when the fundamental, $\theta$, is positive, which is not first-best optimal for the central bank. 


\section{References}

Allen, Franklin, Stephen Morris, and Hyun Song Shin, 2006, Beauty contests and iterated expectations in asset markets, Review of Financial Studies 19, 719-752.

Angeletos, George-Marios, Christian Hellwig, and Alessandro Pavan, 2007, Dynamic global games of regime change: Learning, multiplicity and timing of attacks, Econometrica 75, $711-756$.

Angeletos, George-Marios, Guido Lorenzoni, and Alessandro Pavan, 2007, Wall street and silicon valley: A delicate interaction, MIT working paper.

Angeletos, George-Marios, and Alessandro Pavan, 2007, Efficient use of information and social value of information, Econometrica 75, 1103-1142.

Angeletos, George-Marios, and Ivan Werning, 2006, Crises and prices - information aggregateion, multiplicity and volatility, American Economic Review 96, 1720-36.

Bacchetta, Philippe, and Eric van Wincoop, 2006, Can information heterogeneity explain the exchange rate determination puzzle?, American Economic Review 96, 552-576.

Baker, Malcolm, Jeremy Stein, and Jeffrey Wurgler, 2003, When does the market matter? stock prices and the investment of equity-dependent firms, Quarterly Journal of Economics 118, 969-1006.

Barlevy, Gadi, and Pietro Veronesi, 2000, Information acquisition in financial markets, Review of Economic Studies 67, 79-90.

Battaglini, Marco, and Roland Benabou, 2003, Trust, coordination, and the industrial organization of political activism, Journal of the European Economic Association 1, 851-870.

Bolton, Patrick, Markus Brunnermeier, and Laura Veldkamp, 2007, Leadership, coordination and mission-driven management, Working Paper.

Boot, Arnoud, and Anjan Thakor, 1997, Financial system architecture, Review of Financial Studies 10, 693-733.

Broner, Fernando, 2008, Discrete devaluations and multiple equilibria in a first generation model of currency crises, Journal of Monetary Economics 55, 592-605. 
Bru, Lluis, and Xavier Vives, 2002, Informational externalities, herding and incentives, Journal of Institutional and Theoretical Economics 158, 91-105.

Burnside, Craig, Martin Eichenbaum, and Sergio Rebelo, 2001, Prospective deficits and the asian currency crisis, Journal of Political Economy 109, 1155-1197.

Chen, Qi, Itay Goldstein, and Wei Jiang, 2007, Price informativeness and investment sensitivity to stock price, Review of Finacial Studies 20, 619-650.

Dasgupta, Amil, 2005, Coordination and delay in global games, Journal of Economic Theory forthcoming.

Dow, James, and Gary Gorton, 1997, Stock market efficiency and economic efficiency: Is there a connection?, Journal of Finance 52, 1087-1129.

Drazen, Allan, 2000, Interest-rate and borrowing defense against speculative attack, Carnegie-Rocehster Conference Series on Public Policy 53, 303-348.

Easley, David, Soeren Hvidkjaer, and Maureen O'Hara, 2002, Is information risk a determinant of asset returns?, Journal of Finance 57, 2185-2221.

Eichengreen, Barry, Andrew Rose, and Charles Wyplosz, 1995, Exchange market mayhem: the antecedents and aftermath of speculative attacks, Economic Policy 10, 251-312.

Evans, Martin D. D., and Richard K. Lyons, 2002, Order flow and exchange rate dynamics, Journal of Political Economy 19, 170-180.

Fishman, Mike J., and Kathleen M. Hagerty, 1992, Insider trading and the efficiency of stock prices, RAND Journal of Economics 23, 106-122.

Flood, Robert, and Peter Garber, 1984, Collapsing exchange-rate regimes: Some linear examples, Journal of International Economics 17, 1-13.

Froot, Kenneth, David Scharfstein, and Jeremy Stein, 1992, Herd on the street: informational inefficiencies in a market with short-term speculation, Journal of Finance 47, 1461-1484.

Fulghieri, Paolo, and Dmitry Lukin, 2001, Information production, dilution costs, and optimal security design, Journal of Financial Economics 61, 3-42. 
Goldstein, Itay, and Alexander Guembel, 2008, Manipulation and the allocational role of prices, Review of Economic Studies forthcoming.

Grossman, Sanford, and Joseph Stiglitz, 1980, On the impossibility of informationally efficient markets, American Economic Review 70, 393-408.

Heinemann, Frank, and Camille Cornand, 2004, Optimal degree of public information dissemination, CESifo working paper 1354.

Hellwig, Christian, 2005, Heterogeneous information and the benefits of transparency, UCLA working paper.

, Arijit Mukherji, and Aleh Tsyvinski, 2006, Self-fulfilling currency crises: The role of interest rates, American Economic Review 96, 1769-87.

Hirshleifer, David, Avanidhar Subrahmanyam, and Sheridan Titman, 1994, Security analysis and trading patterns when some investors receive information before others, Journal of Finance 49, 1665-1698.

Ito, Takatoshi, Richard K. Lyons, and Michael T. Melvin, 1998, Is there private information in the foreign exchange market? the tokyo experiment, Journal of Finance 53, 1111-1130.

Khanna, Naveen, Steve L. Slezak, and Michael H. Bradley, 1994, Insider trading, outside search and resource allocation: Why firms and society may disagree on insider trading restrictions, Review of Financial Studies 7, 575-608.

Khanna, Naveen, and Ramana Sonti, 2004, Value creating stock manipulation: Feedback effect of stock prices on firm value, Journal of Financial Markets 7, 237-270.

Krugman, Paul, 1979, A model of self-fulfilling balance of payments crises, Journal of Money, Credit, and Banking 11, 311-25.

Kurlat, Pablo, 2008, Speculative attacks against a strategic agent with incomplete information, MIT Working Paper.

Leland, Hayne, 1992, Insider trading: Should it be prohibited?, Journal of Political Economy $100,859-887$.

Luo, Yuanzhi, 2005, Do insiders learn from outsiders? evidence from mergers and acquisitions, Journal of Finance 60, 1951-1972. 
Lyons, Richard K., 2001, The Microstructure Approach to Exchange Rates (MIT Press: Cambridge, MA).

Morris, Stephen, and Hyun Song Shin, 1998, Unique equilibrium in a model of self-fulfilling currency attacks, American Economic Review 88, 587-597.

— , 2002, The social value of public information, American Economic Review 92, 15211534 .

— 2005, Central bank transparency and the signal value of prices, Brookings Papers on Economic Activity.

Obstfeld, Maurice, 1996, Models of currency crises with self-fulling features, European Economic Review 40, 1037-48.

Ozdenoren, Emre, and Kathy Yuan, 2007, Feedback effects and asset prices, Journal of Finance forthcoming.

Piazzesi, Monika, 2005, Bond yields and the federal reserve, Journal of Political Economy $113,311-344$.

Salant, Stephen W., and Dale W. Henderson, 1978, Market anticipations of government policies and the price of gold, Journal of Political Economy 86, 627-648.

Scharfstein, David, and Jeremy Stein, 1990, Herd behavior and investment, American Economic Review 80, 465-479.

Subrahmanyam, Avanidhar, and Sheridan Titman, 1999, The going-public decision and the development of financial markets, Journal of Finance 54, 1045-1082.

Svensson, Lars, 2005, The social value of public information: Morris and shin (2002) is actually pro transparency, not con, American Economic Review 96, 448-452.

Veldkamp, Laura, 2006a, Information markets and the comovement of asset prices, Review of Economic Studies 73, 823-845.

— 2006b, Media frenzies in markets for financial information, American Economic Review 96, 577-601. 
Woodford, Michael, 2005, Central bank communication and policy effectiveness, 2005 Symposium of the Federal Reserve Bank of Kansas City at Jacksons Hole, The Greenspan Era: Lessons for the Future. 